\title{
Uniqueness conditions for constrained three-way factor decompositions with linearly dependent loadings
}

\author{
Alwin Stegeman* and André L. F. de Almeida ${ }^{\dagger}$
}

September 28, 2009

\begin{abstract}
In this paper, we derive uniqueness conditions for a constrained version of the Parallel Factor (Parafac) decomposition, also known as Canonical decomposition (Candecomp). Candecomp/Parafac (CP) decomposes a three-way array into a prespecified number of outer product arrays. The constraint is that some vectors forming the outer product arrays are linearly dependent according to a prespecified pattern. This is known as the PARALIND family of models. An important subclass is where some vectors forming the outer product arrays are repeated according to a prespecified pattern. These are known as CONFAC decompositions. We discuss the relation between PARALIND, CONFAC and the three-way decompositions CP, Tucker3, and the decomposition in block terms. We provide both essential uniqueness conditions and partial uniqueness conditions for PARALIND and CONFAC, and discuss the relation with uniqueness of constrained Tucker3 models and the block decomposition in rank- $(L, L, 1)$ terms. Our results are demonstrated by means of examples.
\end{abstract}

Keywords: PARALIND, CONFAC, Parafac, Candecomp, tensor decomposition, uniqueness.

AMS subject classifications: 15A69, 62H25

\footnotetext{
${ }^{*}$ Corresponding author. Alwin Stegeman is with the Heymans Institute for Psychological Research, University of Groningen, Grote Kruisstraat 2/1, 9712 TS Groningen, The Netherlands. Phone: ++3150363 6193, fax: ++31 50 363 6304, email: a.w.stegeman@rug.nl, URL: http://www.gmw.rug.nl/ stegeman. Research supported by the Dutch Organisation for Scientific Research (NWO), VENI grant 451-04-102 and VIDI grant 452-08-001.

${ }^{\dagger}$ André L. F. de Almeida is with the I3S Laboratory, University of Nice-Sophia Antipolis (UNSA), CNRS, France. Phone: ++33 492942 736, fax: ++33 492942 896, email: lima@i3s.unice.fr. URL: www.i3s.unice.fr/ lima. Research supported by CAPES/COFECUB postdoctoral fellowship under project 544/07.
} 


\section{Introduction}

Hitchcock $[18,19]$ introduced a generalized rank and related decomposition of a multi-way array or tensor. The same decomposition was proposed independently by Carroll and Chang [7] and Harshman [16] for component analysis of three-way data arrays. They named it Candecomp and Parafac, respectively. We denote the Candecomp/Parafac (CP) model, i.e. the decomposition with a residual term, as

$$
\underline{\mathbf{X}}=\sum_{r=1}^{R}\left(\mathbf{a}_{r} \circ \mathbf{b}_{r} \circ \mathbf{c}_{r}\right)+\underline{\mathbf{E}},
$$

where $\underline{\mathbf{X}}$ is a three-way array of size $I \times J \times K$, ○ denotes the outer product, and $\mathbf{a}_{r}, \mathbf{b}_{r}$ and $\mathbf{c}_{r}$ are vectors of size $I \times 1, J \times 1$ and $K \times 1$, respectively. To find the latter vectors, an iterative algorithm is used which minimizes the Frobenius norm of the residual array $\underline{\mathbf{E}}$. For an overview and comparison of CP algorithms, see Hopke et al. [20] and Tomasi and Bro [44].

The rank of a three-way array $\underline{\mathbf{X}}$ is defined in the usual way, i.e. the smallest number of rank-1 arrays whose sum equals $\underline{\mathbf{X}}$. A three-way array has rank 1 if it is the outer product of three vectors, i.e. $\mathbf{a} \circ \mathbf{b} \circ \mathbf{c}$. It follows that the $\mathrm{CP}$ model tries to find a best rank- $R$ approximation to the three-way array $\underline{\mathbf{X}}$.

The real-valued CP model, i.e. where $\underline{\mathbf{X}}$ and the model parameters are real-valued, was introduced in Psychometrics (Carroll and Chang [7]) and Phonetics (Harshman [16]). Later on, it was also applied in Chemometrics and food industry (Bro [4] and Smilde, Bro and Geladi [32]). For other applications of CP in Psychometrics, see Kroonenberg [25]. Complex-valued applications of $\mathrm{CP}$ occur in Signal Processing, especially wireless telecommunications; see Sidiropoulos, Giannakis and Bro [28], Sidiropoulos, Bro and Giannakis [29] and De Lathauwer and Castaing [13]. Also, $\mathrm{CP}$ describes the basic structure of fourth-order cumulants of multivariate data on which a lot of algebraic methods for Independent Component Analysis are based (Comon [8], De Lathauwer, De Moor and Vandewalle [9], and Hyvärinen, Karhunen and Oja [21]). In this paper, we consider the real-valued CP model. All occurrences of three-way rank are assumed to be over the real field.

For later use, we mention that the CP model (1.1) is a special case of the Tucker3 model of Tucker [45]. The latter is defined as

$$
\underline{\mathbf{X}}=\sum_{r=1}^{R} \sum_{p=1}^{P} \sum_{q=1}^{Q} g_{r p q}\left(\mathbf{a}_{r} \circ \mathbf{b}_{p} \circ \mathbf{c}_{q}\right)+\underline{\mathbf{E}} .
$$

Clearly, the case with $R=P=Q$ and $g_{r p q}=0$ if $(r, p, q) \neq(r, r, r)$ yields (1.1). The $R \times P \times Q$ array $\underline{\mathbf{G}}$ with entries $g_{r p q}$ is referred to as the core array. The matrices $\left[\mathbf{a}_{1}|\ldots| \mathbf{a}_{R}\right],\left[\mathbf{b}_{1}|\ldots| \mathbf{b}_{P}\right]$ 
and $\left[\mathbf{c}_{1}|\ldots| \mathbf{c}_{Q}\right]$ are called the component matrices.

A matrix notation of the CP model (1.1) is as follows. Let $\mathbf{X}_{k}(I \times J)$ and $\mathbf{E}_{k}(I \times J)$ denote the $k$-th frontal slice of $\underline{\mathbf{X}}$ and $\underline{\mathbf{E}}$, respectively. Then (1.1) can be written as

$$
\mathbf{X}_{k}=\mathbf{A ~ C}_{k} \mathbf{B}^{T}+\mathbf{E}_{k}, \quad k=1, \ldots K
$$

where the component matrices $\mathbf{A}(I \times R)$ and $\mathbf{B}(J \times R)$ have the vectors $\mathbf{a}_{r}$ and $\mathbf{b}_{r}$ as columns, respectively, and $\mathbf{C}_{k}(R \times R)$ is the diagonal matrix with the $k$-th elements of the vectors $\mathbf{c}_{r}$ on its diagonal. The model part of the $\mathrm{CP}$ model is characterized by $(\mathbf{A}, \mathbf{B}, \mathbf{C})$, where component matrix $\mathbf{C}(K \times R)$ has the vectors $\mathbf{c}_{r}$ as columns.

The most attractive feature of CP is its uniqueness property. Kruskal [26] has shown that, for fixed residuals $\underline{\mathbf{E}}$, the vectors $\mathbf{a}_{r}, \mathbf{b}_{r}$ and $\mathbf{c}_{r}$ are unique up to rescaling/counterscaling within each triplet $\left(\mathbf{a}_{r}, \mathbf{b}_{r}, \mathbf{c}_{r}\right)$ and a permutation of the order of the triplets if

$$
k_{\mathbf{A}}+k_{\mathbf{B}}+k_{\mathbf{C}} \geq 2 R+2,
$$

where $k_{\mathbf{A}}, k_{\mathbf{B}}, k_{\mathbf{C}}$ denote the k-ranks of the component matrices. The k-rank of a matrix is the largest number $x$ such that every subset of $x$ columns of the matrix is linearly independent. If a $\mathrm{CP}$ solution is unique up to these indeterminacies, it is called essentially unique. Two CP solutions that are identical up to the essential uniqueness indeterminacies, will be called equivalent. A more accessible proof of the uniqueness condition (1.4) can be found in Stegeman and Sidiropoulos [38].

For the case where one of the component matrices $\mathbf{A}, \mathbf{B}$ and $\mathbf{C}$ has full column rank (i.e., rank equal to the number of columns $R$ ), a more relaxed uniqueness condition than (1.4) has been derived by Jiang and Sidiropoulos [22] and De Lathauwer [10]. See also Stegeman, Ten Berge and De Lathauwer [37]. Stegeman [36] shows that this condition is implied by (1.4).

In this paper, we consider a constrained version of the $\mathrm{CP}$ decomposition in which the columns of $\mathbf{A}, \mathbf{B}$ and $\mathbf{C}$ are linearly dependent according to a prespecified pattern. This type of model is introduced in Bro, Harshman, Sidiropoulos and Lundy [6] and previous versions of [6], and is named PARALIND (PARAllel profiles with LINear Dependencies). Instead of (A, B, C), a PARALIND decomposition is characterized by $(\mathbf{A} \boldsymbol{\Psi}, \mathbf{B} \boldsymbol{\Phi}, \mathbf{C} \boldsymbol{\Omega})$, where $\mathbf{A}$ is $I \times R_{1}, \mathbf{B}$ is $J \times R_{2}$, $\mathbf{C}$ is $K \times R_{3}$, $\boldsymbol{\Psi}$ is $R_{1} \times R, \boldsymbol{\Phi}$ is $R_{2} \times R$, and $\boldsymbol{\Omega}$ is $R_{3} \times R$. The prespecified matrices $\boldsymbol{\Psi}, \boldsymbol{\Phi}$ and $\boldsymbol{\Omega}$ contain the patterns of linear dependency of the columns of $\mathbf{A}, \mathbf{B}$ and $\mathbf{C}$, respectively. We refer to $\boldsymbol{\Psi}, \boldsymbol{\Phi}$ and $\Omega$ as the constraint matrices.

In nearly all applications of PARALIND decompositions, the linear dependencies take the form of identical columns. Hence, the columns of $\mathbf{A}, \mathbf{B}$ and $\mathbf{C}$ may be repeated more than once in the 
triplets $\left(\mathbf{a}_{r}, \mathbf{b}_{r}, \mathbf{c}_{r}\right)$ according to a prespecified pattern. The patterns of repetition are given by the constraint matrices $\boldsymbol{\Psi}, \boldsymbol{\Phi}$ and $\boldsymbol{\Omega}$, that contain (possibly identical) unit vectors as columns. The constraint matrices are assumed to have full row rank, which implies $R \geq \max \left(R_{1}, R_{2}, R_{3}\right)$. This assumption guarantees that each column of $\mathbf{A}, \mathbf{B}$ and $\mathbf{C}$ appears at least once in the decomposition. In de Almeida, Favier and Mota [3], this type of decomposition is introduced as CONFAC (CONstrained FACtors).

For given PARALIND or CONFAC constraint matrices, an Alternating Least Squares (ALS) algorithm for finding the component matrices $\mathbf{A}, \mathbf{B}$ and $\mathbf{C}$ that minimize the Frobenius norm of the residual array has been proposed in see Bro et al. [6] and de Almeida et al. [3]. However, analogous to the ALS algorithm for the CP decomposition, it may terminate in a local minimum instead of the global minimum. This fallacy may be overcome by running the algorithm several times with random starting points. A more severe problem that PARALIND and CONFAC may share with $\mathrm{CP}$ is nonexistence of an optimal solution $(\mathbf{A}, \mathbf{B}, \mathbf{C})$. For $\mathrm{CP}$ this problem results in so-called "degenerate solutions"; see Stegeman [33, 34, 35], Krijnen, Dijkstra and Stegeman [24], De Silva and Lim [15] and Stegeman and De Lathauwer [39]. However, in the study of uniqueness of a given PARALIND or CONFAC solution this potential problem does not play a role.

In the sequel, we use the name CONFAC for decompositions in which the constraint matrices have unit vectors as columns, and we use the name PARALIND for decompositions in which the constraint matrices (are allowed to) have more general forms.

As an example of CONFAC, let $R=4, R_{1}=R_{2}=2, R_{3}=3$, and

$$
\boldsymbol{\Psi}=\boldsymbol{\Phi}=\left[\begin{array}{llll}
1 & 1 & 0 & 0 \\
0 & 0 & 1 & 1
\end{array}\right], \quad \boldsymbol{\Omega}=\left[\begin{array}{llll}
1 & 0 & 1 & 0 \\
0 & 1 & 0 & 0 \\
0 & 0 & 0 & 1
\end{array}\right]
$$

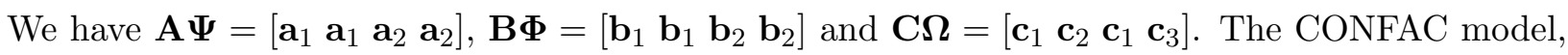
i.e. the decomposition plus the residual term, is then given by

$$
\underline{\mathbf{X}}=\left(\mathbf{a}_{1} \circ \mathbf{b}_{1} \circ \mathbf{c}_{1}\right)+\left(\mathbf{a}_{1} \circ \mathbf{b}_{1} \circ \mathbf{c}_{2}\right)+\left(\mathbf{a}_{2} \circ \mathbf{b}_{2} \circ \mathbf{c}_{1}\right)+\left(\mathbf{a}_{2} \circ \mathbf{b}_{2} \circ \mathbf{c}_{3}\right)+\underline{\mathbf{E}} .
$$

In Bro et al. [6], CONFAC and PARALIND decompositions are used to analyze flow injection data and fluorescence data. In [3], de Almeida et al. exploit the CONFAC structure to design multipleantenna transmissions in the context of wireless telecommunications and signal processing. It is shown that the three constraint matrices $\boldsymbol{\Psi}, \boldsymbol{\Phi}$ and $\boldsymbol{\Omega}$ are design parameters of the transmission system. By varying their patterns of zeros and ones, it is possible to adjust the model parameters 
thus providing some flexibility to the design of the system. From a signal processing and modeling viewpoint, the CONFAC approach of [3] generalizes several related works [30], [31], [14], [1], [2], where the interactions between different factors are either absent, as in [31], or fixed to a prescribed interaction pattern as in [30], [14], [1], [2].

In this paper, we derive uniqueness conditions for one of the component matrices $\mathbf{A}, \mathbf{B}$ and $\mathbf{C}$ of a PARALIND or CONFAC decomposition, for given constraint matrices $\boldsymbol{\Psi}, \boldsymbol{\Phi}$ and $\boldsymbol{\Omega}$. Our results are obtained by applying the approach of Jiang and Sidiropoulos [22] for CP uniqueness to PARALIND. As [22], we prove an essential uniqueness condition for one component matrix by using the Permutation Lemma of Kruskal [26]. Moreover, we extend this result by proving a partial uniqueness condition for one component matrix. For this, we use the Equivalence Lemma for Partitioned Matrices proven by De Lathauwer [11].

In the signal processing applications of the CONFAC decomposition cited above, the uniqueness of one particular component matrix (the one containing an estimate of the transmitted information signal in the telecom system) is most important. This shows that, although our uniqueness results are formulated for a single component matrix, they have immediate practical implications.

The paper is organized as follows. In Section 2, we discuss the relations between PARALIND, CONFAC and other three-way decompositions such as CP, Tucker3, and block decompositions. In Section 3, we discuss and define essential and partial uniqueness of the component matrices. In Sections 4 and 6, we present our essential and partial PARALIND uniqueness results, respectively. Sections 5 and 7 illustrate our uniqueness results by means of various examples. In Section 8 , we discuss the relation between our approach and uniqueness of Tucker3 models with a constrained core array. PARALIND can be written into the latter form, as will be seen in Section 2. In Section 9, we consider uniqueness for the block decomposition in rank- $(L, L, 1)$ terms, which is a special case of CONFAC. Finally, Section 10 contains a discussion of our findings.

\section{CONFAC, PARALIND and other three-way decompositions}

Here, we discuss the relations between CONFAC, PARALIND and other three-way decompositions. First, we observe that if $\boldsymbol{\Psi}=\boldsymbol{\Phi}=\boldsymbol{\Omega}=\mathbf{I}_{R}$, then the decomposition is identical to CP. But there are more cases where this is true. These are formulated in the following lemma.

Lemma 2.1 Consider a CONFAC model with $R_{1}=R_{2}=R_{3}$ and $\boldsymbol{\Psi}=\boldsymbol{\Pi}_{1} \boldsymbol{\Phi}=\boldsymbol{\Pi}_{2} \boldsymbol{\Omega}$, with $\boldsymbol{\Pi}_{1}$ 
and $\boldsymbol{\Pi}_{2}$ permutation matrices. Then the CONFAC model can be written in the following CP form:

$$
\underline{\mathbf{X}}=\sum_{r=1}^{R_{1}} n_{r}\left(\mathbf{a}_{r} \circ \mathbf{b}_{\pi_{1}(r)} \circ \mathbf{c}_{\pi_{2}(r)}\right)+\underline{\mathbf{E}},
$$

where $n_{r}$ denotes the row sum of row $r$ of $\boldsymbol{\Psi}$, and $\pi_{1}(\cdot)$ and $\pi_{2}(\cdot)$ are the row permutations of $\mathbf{\Phi}$ and $\boldsymbol{\Omega}$ corresponding to $\boldsymbol{\Pi}_{1}$ and $\boldsymbol{\Pi}_{2}$, respectively.

Proof. The component matrices all have the same number of columns, since $R_{1}=R_{2}=R_{3}$. The condition $\boldsymbol{\Psi}=\boldsymbol{\Pi}_{1} \boldsymbol{\Phi}=\boldsymbol{\Pi}_{2} \boldsymbol{\Omega}$ implies that each triplet of vectors forming a rank-1 array in the decomposition does not share a vector with another triplet. Moreover, the triplet containing $\mathbf{a}_{r}$ is repeated as often as the number of times $\mathbf{a}_{r}$ appears in $\mathbf{A} \boldsymbol{\Psi}$, which is equal to the row sum of row $r$ of $\boldsymbol{\Psi}$. Hence, there are $R_{1}$ triplets and triplet $r$ is repeated $n_{r}$ times. This completes the proof. $\square$

Note that Lemma 2.1 does not cover all cases where CONFAC reduces to CP. For example, let $R_{1}=3, R_{2}=R_{3}=2, R=3, \Psi=\mathbf{I}_{3}$, and

$$
\boldsymbol{\Phi}=\boldsymbol{\Omega}=\left[\begin{array}{lll}
1 & 1 & 0 \\
0 & 0 & 1
\end{array}\right] .
$$

Then the CONFAC model is given by

$$
\begin{aligned}
\underline{\mathbf{X}} & =\left(\mathbf{a}_{1} \circ \mathbf{b}_{1} \circ \mathbf{c}_{1}\right)+\left(\mathbf{a}_{2} \circ \mathbf{b}_{1} \circ \mathbf{c}_{1}\right)+\left(\mathbf{a}_{3} \circ \mathbf{b}_{2} \circ \mathbf{c}_{2}\right)+\underline{\mathbf{E}} \\
& =\left(\left(\mathbf{a}_{1}+\mathbf{a}_{2}\right) \circ \mathbf{b}_{1} \circ \mathbf{c}_{1}\right)+\left(\mathbf{a}_{3} \circ \mathbf{b}_{2} \circ \mathbf{c}_{2}\right)+\underline{\mathbf{E}},
\end{aligned}
$$

where the latter has the form of the CP model (1.1) with $R=2$.

Next, we discuss the relation between PARALIND, CONFAC and the Tucker3 model (1.2). De Almeida et al. [3] show that a PARALIND model can be written as a Tucker3 model with $R_{1} \times R_{2} \times R_{3}$ core array

$$
\underline{\mathbf{G}}=\sum_{r=1}^{R}\left(\boldsymbol{\psi}_{r} \circ \boldsymbol{\phi}_{r} \circ \boldsymbol{\omega}_{r}\right),
$$

where $\boldsymbol{\psi}_{r}, \phi_{r}$ and $\boldsymbol{\omega}_{r}$ are the $r$-th columns of $\boldsymbol{\Psi}, \boldsymbol{\Phi}$ and $\boldsymbol{\Omega}$, respectively. Hence, the core array $\underline{\mathbf{G}}$ satisfies a CP decomposition with component matrices $\boldsymbol{\Psi}, \boldsymbol{\Phi}$ and $\boldsymbol{\Omega}$. For a CONFAC decomposition, it can be seen that $\underline{\mathbf{G}}$ contains at most $R$ nonzero entries. Hence, CONFAC is equivalent to a Tucker3 model in which the constrained core array has a fixed pattern of zeros and integer-valued nonzeros. If $g_{r p q}=n$, then the triplet $\left(\mathbf{a}_{r}, \mathbf{b}_{p}, \mathbf{c}_{q}\right)$ is contained $n$ times in the decomposition. Constrained Tucker3 models have applications in Chemometrics, see Smilde et al. [32]. 
There also exist three-way decompositions that are hybrid forms of CP and Tucker3. Some of these may also be written in PARALIND or CONFAC form. This derivation is beyond the scope of this paper, however. We refer to Harshman and Lundy [17] and Bro [5] for an overview of these hybrid models.

A third decomposition related to PARALIND and CONFAC is the decomposition in block terms, introduced by De Lathauwer [12]. Before we discuss this decomposition, we need to introduce some notions. A mode- $n$ vector of an $I_{1} \times I_{2} \times I_{3}$ array is an $I_{n} \times 1$ vector obtained from the array by varying the $n$-th index and keeping the other indices fixed. The mode- $n$ rank is defined as the dimension of the subspace spanned by the mode- $n$ vectors of the array. When a three-way array has mode-1 rank $L$, mode- $2 \operatorname{rank} M$, and mode- $3 \operatorname{rank} N$, it is said to be $\operatorname{rank}(L, M, N)$. The mode- $n$ rank generalizes the row and column rank of matrices. Note that a rank- $(1,1,1)$ array has rank 1 and vice versa.

The decomposition in block terms of [12] is a generalization of $\mathrm{CP}$ in which the array is not decomposed into rank-1 arrays but into rank- $(L, M, N)$ arrays, where we denote the number of terms as $F$. Term $f$ in the decomposition can be written in Tucker3 form with an $L \times M \times N$ core array $\underline{\mathbf{G}}^{(f)}$ that is rank- $(L, M, N)$, and matrices $\mathbf{A}_{f}(I \times L), \mathbf{B}_{f}(J \times M)$, and $\mathbf{C}_{f}(K \times N)$ that have full column rank.

De Lathauwer [12] discusses the decomposition in $\operatorname{rank}-(L, L, 1)$ terms as a special case. By absorbing the $L \times L \times 1$ core arrays $\underline{\mathbf{G}}^{(f)}$ into $\mathbf{A}_{f}$, it can be written as

$$
\begin{aligned}
\underline{\mathbf{X}} & =\sum_{f=1}^{F}\left(\mathbf{A}_{f} \mathbf{B}_{f}^{T}\right) \circ \mathbf{c}_{f}+\underline{\mathbf{E}} \\
& =\sum_{f=1}^{F}\left(\sum_{l=1}^{L}\left(\mathbf{a}_{l}^{(f)} \circ \mathbf{b}_{l}^{(f)}\right)\right) \circ \mathbf{c}_{f}+\underline{\mathbf{E}} \\
& =\sum_{f=1}^{F} \sum_{l=1}^{L}\left(\mathbf{a}_{l}^{(f)} \circ \mathbf{b}_{l}^{(f)} \circ \mathbf{c}_{f}\right)+\underline{\mathbf{E}},
\end{aligned}
$$

where $\mathbf{a}_{l}^{(f)}$ and $\mathbf{b}_{l}^{(f)}$ are the $l$-th columns of $\mathbf{A}_{f}$ and $\mathbf{B}_{f}$, respectively. It can be seen that (2.5) is the CONFAC model with $\mathbf{A}=\left[\mathbf{A}_{1}|\ldots| \mathbf{A}_{F}\right], \mathbf{B}=\left[\mathbf{B}_{1}|\ldots| \mathbf{B}_{F}\right], \mathbf{C}=\left[\mathbf{c}_{1} \ldots \mathbf{c}_{F}\right], \mathbf{\Psi}=\mathbf{\Phi}=\mathbf{I}_{L F}$, and $\boldsymbol{\Omega}=\mathbf{I}_{F} \otimes \mathbf{1}_{L}^{T}$, where $\mathbf{1}_{L}$ is an $L \times 1$ vectors of ones, and $\otimes$ denotes the Kronecker product.

The relation between PARALIND, CONFAC and the general decomposition in rank- $(L, M, N)$ terms is more complicated. In order to obtain a PARALIND or CONFAC form, each core array $\underline{\mathbf{G}}^{(f)}$ must be transformed by nonsingular transformations into some canonical form. The inverses 
of the transformations can be absorbed into $\mathbf{A}_{f}, \mathbf{B}_{f}$, and $\mathbf{C}_{f}$. Whether the resulting decomposition obeys the PARALIND or CONFAC structure depends on the canonical forms of the core arrays. This can be illustrated by considering the case $L=M=N=2$, for which the canonical forms are given by De Silva and Lim [15]. Hence, the core arrays are $2 \times 2 \times 2$ and rank-(2,2,2). Each core array satisfies one of the following: it is generic and has rank 2, it is generic and has rank 3 , or it is degenerate and has rank 3 . If the core array $\underline{\mathbf{G}}^{(f)}$ is degenerate and has rank 3 , then it can be transformed to a canonical form such that term $f$ of the decomposition is

$$
\left(\mathbf{a}_{1}^{(f)} \circ \mathbf{b}_{1}^{(f)} \circ \mathbf{c}_{1}^{(f)}\right)+\left(\mathbf{a}_{2}^{(f)} \circ \mathbf{b}_{2}^{(f)} \circ \mathbf{c}_{1}^{(f)}\right)+\left(\mathbf{a}_{1}^{(f)} \circ \mathbf{b}_{2}^{(f)} \circ \mathbf{c}_{2}^{(f)}\right) .
$$

It can be checked that this term has the CONFAC structure. If the core array $\underline{\mathbf{G}}^{(f)}$ is generic and has rank 2 , then it can be transformed to the a diagonal canonical form such that term $f$ of the decomposition is $\left(\mathbf{a}_{1}^{(f)} \circ \mathbf{b}_{1}^{(f)} \circ \mathbf{c}_{1}^{(f)}\right)+\left(\mathbf{a}_{2}^{(f)} \circ \mathbf{b}_{2}^{(f)} \circ \mathbf{c}_{2}^{(f)}\right)$. This is of CP form, and, hence, of CONFAC form. If the core array $\underline{\mathbf{G}}^{(f)}$ is generic and has rank 3 , then it can be transformed to a canonical form such that term $f$ of the decomposition is

$$
\left(\mathbf{a}_{1}^{(f)} \circ \mathbf{b}_{1}^{(f)} \circ \mathbf{c}_{1}^{(f)}\right)+\left(\mathbf{a}_{2}^{(f)} \circ \mathbf{b}_{2}^{(f)} \circ \mathbf{c}_{1}^{(f)}\right)+\left(\mathbf{a}_{1}^{(f)} \circ \mathbf{b}_{2}^{(f)} \circ \mathbf{c}_{2}^{(f)}\right)-\left(\mathbf{a}_{2}^{(f)} \circ \mathbf{b}_{1}^{(f)} \circ \mathbf{c}_{2}^{(f)}\right) \text {. }
$$

This term is of PARALIND form but does not have CONFAC structure. We conjecture that $\underline{\mathbf{G}}^{(f)}$ cannot be transformed to a canonical form such that term $f$ of the decomposition is of CONFAC form. Since term $f$ of the decomposition only involves columns from $\mathbf{A}_{f}, \mathbf{B}_{f}$ and $\mathbf{C}_{f}$, the complete decomposition can be written in CONFAC form when the core arrays are either degenerate and have rank 3 , or generic and have rank 2 .

\section{Essential and partial uniqueness in PARALIND}

Before we present our uniqueness results for the component matrices in PARALIND, we discuss the meaning of essential and partial uniqueness in PARALIND. Recall that essential uniqueness in $\mathrm{CP}$ holds when the triplets $\left(\mathbf{a}_{r}, \mathbf{b}_{r}, \mathbf{c}_{r}\right)$ are unique up to scaling/counterscaling within each triplet, and a permutation of the order of the triplets. More formally, if we have an essentially unique $\mathrm{CP}$ solution $(\mathbf{A}, \mathbf{B}, \mathbf{C})$ and an alternative solution $(\overline{\mathbf{A}}, \overline{\mathbf{B}}, \overline{\mathbf{C}})$ with the same residuals, then $\overline{\mathbf{A}}=\mathbf{A} \boldsymbol{\Pi} \boldsymbol{\Lambda}_{a}, \overline{\mathbf{B}}=\mathbf{B} \boldsymbol{\Pi} \boldsymbol{\Lambda}_{b}$, and $\overline{\mathbf{C}}=\mathbf{C} \boldsymbol{\Pi} \boldsymbol{\Lambda}_{c}$, where $\boldsymbol{\Pi}$ is a permutation matrix and $\boldsymbol{\Lambda}_{a}, \boldsymbol{\Lambda}_{b}$, and $\boldsymbol{\Lambda}_{c}$ are nonsingular diagonal matrices such that $\boldsymbol{\Lambda}_{a} \boldsymbol{\Lambda}_{b} \boldsymbol{\Lambda}_{c}=\mathbf{I}_{R}$.

In PARALIND, due to the linear dependence of the vectors in the triplets $\left(\mathbf{a}_{r}, \mathbf{b}_{p}, \mathbf{c}_{q}\right)$, there is less freedom of scaling/counterscaling within the triplets without affecting the residuals. Also, 
permuting the order of the triplets may not be possible without affecting the residuals. The PARALIND solutions $(\mathbf{A} \Psi, \mathbf{B} \boldsymbol{\Phi}, \mathbf{C} \boldsymbol{\Omega})$ and $(\mathbf{A} \boldsymbol{\Psi} \Pi, \mathbf{B} \boldsymbol{\Phi} \Pi, \mathbf{C} \boldsymbol{\Omega} \Pi)$ do have the same residuals for a permutation matrix $\boldsymbol{\Pi}$, but the latter solution features constraint matrices that may differ from those in the former solution. Note that we consider a PARALIND solution as a triplet of component matrices corresponding to a fixed triplet of constraint matrices.

To avoid these complications, we define essential uniqueness for one component matrix instead of all three of them together. In particular, we define the following.

Definition 3.1 Let $(\mathbf{A} \boldsymbol{\Psi}, \mathbf{B} \boldsymbol{\Phi}, \mathbf{C} \boldsymbol{\Omega})$ be a PARALIND solution for fixed constraint matrices $\mathbf{\Psi}, \mathbf{\Phi}$, and $\boldsymbol{\Omega}$. If any alternative PARALIND solution $(\overline{\mathbf{A}} \boldsymbol{\Psi}, \overline{\mathbf{B}} \boldsymbol{\Phi}, \overline{\mathbf{C}} \boldsymbol{\Omega})$ with the same residuals satisfies $\overline{\mathbf{A}}=\mathbf{A} \boldsymbol{\Pi} \boldsymbol{\Lambda}$ for some permutation matrix $\boldsymbol{\Pi}$ and some nonsingular diagonal matrix $\boldsymbol{\Lambda}$, then we call A essentially unique.

Note that if $\mathbf{A}$ is essentially unique, then also $\mathbf{A \Pi}$ is essentially unique for any permutation matrix $\boldsymbol{\Pi}$. Hence, the essential uniqueness of the PARALIND component matrices is invariant under row permutations of the constraint matrices $\boldsymbol{\Psi}, \boldsymbol{\Phi}$, and $\boldsymbol{\Omega}$.

Next, we discuss the concept of partial uniqueness. For CP, this term has been used to describe cases where some columns of a component matrix are identified up to their linear span only, or where only a finite number of alternative CP solutions are available (up to $\mathrm{CP}$ essential uniqueness); see Ten Berge [42]. For PARALIND, we adopt the first definition, and we call A partially unique if its columns can be partitioned into disjoint subsets and each subset is identified up to its linear span. This is in line with the discussion on partial uniqueness in PARALIND by Bro et al. [6].

Definition 3.2 Let $(\mathbf{A} \boldsymbol{\Psi}, \mathbf{B} \boldsymbol{\Phi}, \mathbf{C} \boldsymbol{\Omega})$ be a PARALIND solution for fixed constraint matrices $\mathbf{\Psi}, \mathbf{\Phi}$, and $\boldsymbol{\Omega}$. Let the columns of $\mathbf{A}$ be partitioned into disjoint subsets as $\mathbf{A} \boldsymbol{\Pi}_{a}=\left[\mathbf{A}_{1}|\ldots| \mathbf{A}_{F}\right]$, where $\mathbf{\Pi}_{a}$ is a permutation matrix. Suppose that, for any alternative PARALIND solution $(\overline{\mathbf{A}} \boldsymbol{\Psi}, \overline{\mathbf{B}} \boldsymbol{\Phi}, \overline{\mathbf{C}} \boldsymbol{\Omega})$ with the same residuals and with $\overline{\mathbf{A}}$ partitioned as $\overline{\mathbf{A}} \boldsymbol{\Pi}_{a}=\left[\overline{\mathbf{A}}_{1}|\ldots| \overline{\mathbf{A}}_{F}\right]$, it holds that $\overline{\mathbf{A}} \mathbf{\Pi}_{a}=$ $\mathbf{A} \boldsymbol{\Pi}_{a} \boldsymbol{\Pi} \boldsymbol{\Lambda}$, where $\boldsymbol{\Pi}$ is a unique block-permutation matrix, $\boldsymbol{\Lambda}$ is a unique nonsingular block-diagonal matrix, and the block-transformation $\boldsymbol{\Pi} \boldsymbol{\Lambda}$ is compatible with the partition of $\mathbf{A} \boldsymbol{\Pi}_{a}$ and $\overline{\mathbf{A}} \boldsymbol{\Pi}_{a}$. Then we call A partially unique.

Definition 3.2 states that each subset of columns of $\overline{\mathbf{A}} \boldsymbol{\Pi}_{a}$ satisfies $\overline{\mathbf{A}}_{f}=\mathbf{A}_{\pi(f)} \mathbf{S}$, where $\mathbf{S}$ is a unique nonsingular matrix and the permutation $\pi(\cdot)$ is defined by the unique block-permutation 
П. Hence, each subset $\mathbf{A}_{f}$ is identified up to its linear span. Note that when checking partial uniqueness of $\mathbf{A}$ one is free to choose a suitable column permutation $\boldsymbol{\Pi}_{a}$ and partition of $\mathbf{A} \boldsymbol{\Pi}_{a}$.

For the block decomposition in $\operatorname{rank}-(L, M, N)$ terms De Lathauwer [12] defines "essential uniqueness" as the case where the blocks $\mathbf{A}_{f}, \mathbf{B}_{f}$, and $\mathbf{C}_{f}$ in the component matrices $\mathbf{A}=$ $\left[\mathbf{A}_{1}|\ldots| \mathbf{A}_{F}\right], \mathbf{B}=\left[\mathbf{B}_{1}|\ldots| \mathbf{B}_{F}\right], \mathbf{C}=\left[\mathbf{C}_{1}|\ldots| \mathbf{C}_{F}\right]$ are identified up to their linear spans and a simultaneous permutation of the $F$ blocks.

Next, we present an identifiability condition for the PARALIND component matrices due to de Almeida et al. [3]. Let the three matricized forms of the PARALIND core array (2.4) be

$$
\mathbf{G}_{1}=(\boldsymbol{\Phi} \odot \boldsymbol{\Omega}) \boldsymbol{\Psi}^{T} \quad \mathbf{G}_{2}=(\boldsymbol{\Omega} \odot \boldsymbol{\Psi}) \boldsymbol{\Phi}^{T} \quad \mathbf{G}_{3}=(\boldsymbol{\Psi} \odot \boldsymbol{\Phi}) \boldsymbol{\Omega}^{T}
$$

where $\odot$ denotes the Khatri-Rao product, i.e. the column-wise Kronecker product.

Proposition 3.3 Let $(\mathbf{A} \boldsymbol{\Psi}, \mathbf{B} \boldsymbol{\Phi}, \mathbf{C} \boldsymbol{\Omega})$ be a PARALIND solution for fixed constraint matrices $\mathbf{\Psi}$, $\boldsymbol{\Phi}$, and $\boldsymbol{\Omega}$. Let $(\overline{\mathbf{A}} \boldsymbol{\Psi}, \overline{\mathbf{B}} \boldsymbol{\Phi}, \overline{\mathbf{C}} \boldsymbol{\Omega})$ be an alternative PARALIND solution with the same residuals.

(i) If $\mathbf{A}$ and $(\mathbf{B} \otimes \mathbf{C}) \mathbf{G}_{1}$ have full column rank, then $\overline{\mathbf{A}}=\mathbf{A} \mathbf{S}$ for some nonsingular matrix $\mathbf{S}$.

(ii) If $\mathbf{B}$ and $(\mathbf{C} \otimes \mathbf{A}) \mathbf{G}_{2}$ have full column rank, then $\overline{\mathbf{B}}=\mathbf{B} \mathbf{T}$ for some nonsingular matrix $\mathbf{T}$.

(iii) If $\mathbf{C}$ and $(\mathbf{A} \otimes \mathbf{B}) \mathbf{G}_{3}$ have full column rank, then $\overline{\mathbf{C}}=\mathbf{C} \mathbf{U}$ for some nonsingular matrix $\mathbf{U}$.

Proof. We prove only $(i)$. The proofs of $(i i)$ and (iii) follow analogously by interchanging the roles of $(\mathbf{A}, \boldsymbol{\Psi}),(\mathbf{B}, \boldsymbol{\Phi})$ and $(\mathbf{C}, \boldsymbol{\Omega})$.

The structural part of the $J K \times I$ matrix unfolding of the PARALIND model can be written as

$$
((\mathbf{B} \boldsymbol{\Phi}) \odot(\mathbf{C} \boldsymbol{\Omega}))(\mathbf{A} \boldsymbol{\Psi})^{T}=(\mathbf{B} \otimes \mathbf{C}) \mathbf{G}_{1} \mathbf{A}^{T} .
$$

Equating the structural parts of the two PARALIND solutions, we obtain

$$
(\mathbf{B} \otimes \mathbf{C}) \mathbf{G}_{1} \mathbf{A}^{T}=(\overline{\mathbf{B}} \otimes \overline{\mathbf{C}}) \mathbf{G}_{1} \overline{\mathbf{A}}^{T}
$$

Since $(\mathbf{B} \otimes \mathbf{C}) \mathbf{G}_{1}$ has full column rank, it follows that the columns of $\mathbf{A}$ lie in the column space of $\overline{\mathbf{A}}$, i.e. $\mathbf{A}=\overline{\mathbf{A}} \mathbf{S}$ for some square matrix $\mathbf{S}$. Moreover, since $\mathbf{A}$ has full column rank, this implies that also $\overline{\mathbf{A}}$ has full column rank. Hence, $\mathbf{S}$ is nonsingular and $\overline{\mathbf{A}}=\mathbf{A} \mathbf{S}^{-1}$. This completes the proof of $(i)$. 
Note that, in $(i)$ of Proposition 3.3, the matrix $(\mathbf{B} \otimes \mathbf{C}) \mathbf{G}_{1}$ has full column rank if $\mathbf{B}, \mathbf{C}$, and $\mathbf{G}_{1}$ have full column rank. Hence, all component matrices are identified up to their linear span if all of $\mathbf{A}, \mathbf{B}, \mathbf{C}, \mathbf{G}_{1}, \mathbf{G}_{2}$, and $\mathbf{G}_{3}$ have full column rank.

We conclude this section with an invariance result of essential and partial uniqueness, which is useful when checking uniqueness. The result is an adaptation of Ten Berge and Sidiropoulos [40, p.401].

Lemma 3.4 Let $(\mathbf{A} \mathbf{\Psi}, \mathbf{B} \boldsymbol{\Phi}, \mathbf{C} \boldsymbol{\Omega})$ be a PARALIND solution for fixed constraint matrices $\mathbf{\Psi}$, $\mathbf{\Phi}$, and $\boldsymbol{\Omega}$. If $\mathbf{A}$ and $(\mathbf{B} \otimes \mathbf{C}) \mathbf{G}_{1}$ have full column rank, then $\mathbf{A}$ is essentially/partially unique if and only if $\mathbf{I}_{R_{1}}$ is essentially/partially unique in the PARALIND solution $\left(\mathbf{I}_{R_{1}} \boldsymbol{\Psi}, \mathbf{B} \mathbf{\Phi}, \mathbf{C} \boldsymbol{\Omega}\right)$.

Proof. The uniqueness properties of $\mathbf{A}$ are the same for $\mathbf{S A}$, where $\mathbf{S}$ is nonsingular. Consider $\mathbf{S}$ with $\mathbf{S A}=\left[\begin{array}{c}\mathbf{I}_{R_{1}} \\ \mathbf{O}\end{array}\right]$, where $\mathbf{O}$ denotes an all-zero matrix. The all-zero rows of $\mathbf{S A}$ do not affect the uniqueness properties of $\mathbf{S A}$. Indeed, let $\left(\mathbf{I}_{R_{1}} \boldsymbol{\Psi}, \mathbf{B} \boldsymbol{\Phi}, \mathbf{C} \boldsymbol{\Omega}\right)$ have an alternative $(\mathbf{F} \boldsymbol{\Psi}, \overline{\mathbf{B}} \boldsymbol{\Phi}, \overline{\mathbf{C}} \boldsymbol{\Omega})$. Then, as in (3.3),

$$
(\mathbf{B} \otimes \mathbf{C}) \mathbf{G}_{1} \mathbf{I}_{R_{1}}=(\overline{\mathbf{B}} \otimes \overline{\mathbf{C}}) \mathbf{G}_{1} \mathbf{F}^{T}
$$

Full column rank of $(\mathbf{B} \otimes \mathbf{C}) \mathbf{G}_{1}$ implies that also $(\overline{\mathbf{B}} \otimes \overline{\mathbf{C}}) \mathbf{G}_{1}$ has full column rank. Hence,

$$
(\mathbf{B} \otimes \mathbf{C}) \mathbf{G}_{1}\left[\mathbf{I}_{R_{1}} \mathbf{O}\right]=(\overline{\mathbf{B}} \otimes \overline{\mathbf{C}}) \mathbf{G}_{1} \overline{\mathbf{A}}^{T}
$$

implies that the last $I-R_{1}$ rows of $\overline{\mathbf{A}}$ are all-zero. This shows that the uniqueness properties of SA solely depend on its nonzero rows.

\section{Essential uniqueness results for PARALIND}

Here, we discuss and prove essential uniqueness conditions for PARALIND. Since PARALIND is a Tucker3 model with constrained core array (2.4), studying PARALIND uniqueness is analogous to studying uniqueness of Tucker3 models with a constrained core array. Suppose we work under the conditions of Proposition 3.3 and $\mathbf{A}, \mathbf{B}$, and $\mathbf{C}$ are identified up to their linear spans. Equating the $J K \times I$ matricized form of the structural part of the PARALIND model for the original and alternative solutions (see (3.3)) yields

$$
(\mathbf{B} \otimes \mathbf{C}) \mathbf{G}_{1} \mathbf{A}^{T}=(\mathbf{B} \otimes \mathbf{C})(\mathbf{T} \otimes \mathbf{U}) \mathbf{G}_{1} \mathbf{S}^{T} \mathbf{A}^{T}
$$


Since $\mathbf{A}, \mathbf{B}$, and $\mathbf{C}$ have full column rank, this is equivalent to

$$
\mathbf{G}_{1}=(\mathbf{T} \otimes \mathbf{U}) \mathbf{G}_{1} \mathbf{S}^{T}
$$

Equation (4.2) states that the PARALIND core array (2.4) must be invariant under the transformations $\mathbf{S}, \mathbf{T}$, and $\mathbf{U}$ that are applied "on its three sides". If (4.2) implies that $\mathbf{S}$ is a rescaled permutation matrix, then $\mathbf{A}$ is essentially unique. This method of proving essential uniqueness depends on the structure of $\mathbf{G}_{1}$ and can be relatively easy or rather complicated: compare e.g. Ten Berge and Smilde [41], Ten Berge [43] and Kiers, Ten Berge and Rocci [23]. Below, we present a unified approach to essential uniqueness in PARALIND that yields an identical sufficient uniqueness condition for each triplet of constraint matrices $(\boldsymbol{\Psi}, \boldsymbol{\Phi}, \boldsymbol{\Omega})$. By using Kruskal's Permutation Lemma [26], the uniqueness of one component matrix can be obtained separate from the other two component matrices. A more detailed comparison between our approach and uniqueness results for constrained Tucker3 models is contained in Section 8.

Our PARALIND essential uniqueness condition is featured in Section 4.1. In Section 4.2 we discuss necessary uniqueness conditions for CONFAC and PARALIND.

\subsection{An essential uniqueness condition for PARALIND}

Here, we present our essential uniqueness condition for one component matrix in PARALIND. Without loss of generality, we focus on the essential uniqueness of $\mathbf{A}$. To prove our uniqueness condition, we use the approach of Jiang and Sidiropoulos [22] for CP uniqueness. As the latter authors, we make use of Kruskal's Permutation Lemma [26] which is the cornerstone of the proof of Kruskal's uniqueness condition (1.4) for CP. The Permutation Lemma is formulated as follows. Let $\omega(\cdot)$ denote the number of nonzero elements of a vector.

Lemma 4.1 (Permutation Lemma) Let $\mathbf{A}$ and $\overline{\mathbf{A}}$ be two $I \times R_{1}$ matrices and let $k_{\mathbf{A}} \geq 2$. Suppose the following condition holds: for any vector $\mathbf{x}$ such that $\omega\left(\overline{\mathbf{A}}^{T} \mathbf{x}\right) \leq R_{1}-\operatorname{rank}(\overline{\mathbf{A}})+1$, we have $\omega\left(\mathbf{A}^{T} \mathbf{x}\right) \leq \omega\left(\overline{\mathbf{A}}^{T} \mathbf{x}\right)$. Then there exists a unique permutation matrix $\boldsymbol{\Pi}$ and a unique nonsingular diagonal matrix $\boldsymbol{\Lambda}$ such that $\overline{\mathbf{A}}=\mathbf{A} \Pi \mathbf{\Lambda}$.

Our essential uniqueness condition for PARALIND is given in Theorem 4.2 below. Let

$$
N^{*}=\max _{j=1, \ldots, R_{1}}\left(\operatorname{rank}\left(\boldsymbol{\Phi} \operatorname{diag}\left(\boldsymbol{\psi}_{j}^{T}\right) \boldsymbol{\Omega}^{T}\right)\right)
$$


where $\boldsymbol{\psi}_{j}^{T}$ denotes row $j$ of $\boldsymbol{\Psi}$. Hence, $N^{*}$ is the maximum of the ranks of the $R_{1}$ horizontal slices of the core array in (2.4).

Theorem 4.2 Let $(\mathbf{A} \boldsymbol{\Psi}, \mathbf{B} \boldsymbol{\Phi}, \mathbf{C} \boldsymbol{\Omega})$ be a PARALIND solution for fixed constraint matrices $\mathbf{\Psi}, \mathbf{\Phi}$, and $\boldsymbol{\Omega}$. Let $(\overline{\mathbf{A}} \boldsymbol{\Psi}, \overline{\mathbf{B}} \mathbf{\Phi}, \overline{\mathbf{C}} \boldsymbol{\Omega})$ be an alternative PARALIND solution with the same residuals. Suppose $(\mathbf{B} \otimes \mathbf{C}) \mathbf{G}_{1}$ and $\mathbf{A}$ have full column rank. If for any vector $\mathbf{d}$,

$$
\operatorname{rank}\left(\mathbf{B} \boldsymbol{\Phi} \operatorname{diag}\left(\mathbf{\Psi}^{T} \mathbf{d}\right) \boldsymbol{\Omega}^{T} \mathbf{C}^{T}\right) \leq N^{*} \quad \text { implies } \quad \omega(\mathbf{d}) \leq 1
$$

then there exists a unique permutation matrix $\boldsymbol{\Pi}$ and a unique nonsingular diagonal matrix $\boldsymbol{\Lambda}$ such that $\overline{\mathbf{A}}=\mathbf{A} \Pi \mathbf{\Lambda}$.

Proof. The result follows if we show that the conditions of the theorem imply the condition of the Permutation Lemma: for any vector $\mathbf{x}$ such that $\omega\left(\overline{\mathbf{A}}^{T} \mathbf{x}\right) \leq R_{1}-\operatorname{rank}(\overline{\mathbf{A}})+1$, we have $\omega\left(\mathbf{A}^{T} \mathbf{x}\right) \leq \omega\left(\overline{\mathbf{A}}^{T} \mathbf{x}\right)$. As in the proof of $(i)$ of Proposition 3.3, the conditions of the theorem imply that $\overline{\mathbf{A}}$ has full column rank. Hence, the condition of the Permutation Lemma becomes: for any vector $\mathbf{x}$ such that $\omega\left(\overline{\mathbf{A}}^{T} \mathbf{x}\right) \leq 1$, we have $\omega\left(\mathbf{A}^{T} \mathbf{x}\right) \leq \omega\left(\overline{\mathbf{A}}^{T} \mathbf{x}\right)$. For any $\mathbf{x}$, we have

$$
(\mathbf{B} \otimes \mathbf{C}) \mathbf{G}_{1} \mathbf{A}^{T} \mathbf{x}=(\overline{\mathbf{B}} \otimes \overline{\mathbf{C}}) \mathbf{G}_{1} \overline{\mathbf{A}}^{T} \mathbf{x} .
$$

Suppose $\omega\left(\overline{\mathbf{A}}^{T} \mathbf{x}\right)=0$. Then the right-hand side of (4.5) equals the all-zero vector. Since $(\mathbf{B} \otimes \mathbf{C}) \mathbf{G}_{1}$ has full column rank, it follows that also $\mathbf{A}^{T} \mathbf{x}$ is all-zero. Hence, $\omega\left(\overline{\mathbf{A}}^{T} \mathbf{x}\right)=0$ implies $\omega\left(\mathbf{A}^{T} \mathbf{x}\right)=0$.

It remains to show that $\omega\left(\overline{\mathbf{A}}^{T} \mathbf{x}\right)=1$ implies $\omega\left(\mathbf{A}^{T} \mathbf{x}\right) \leq 1$. Rewriting (4.5) in $J \times K$ matrix form, we obtain

$$
\mathbf{B} \boldsymbol{\Phi} \operatorname{diag}\left(\mathbf{\Psi}^{T} \mathbf{A}^{T} \mathbf{x}\right) \boldsymbol{\Omega}^{T} \mathbf{C}^{T}=\overline{\mathbf{B}} \boldsymbol{\Phi} \operatorname{diag}\left(\boldsymbol{\Psi}^{T} \overline{\mathbf{A}}^{T} \mathbf{x}\right) \boldsymbol{\Omega}^{T} \overline{\mathbf{C}}^{T}
$$

Suppose $\omega\left(\overline{\mathbf{A}}^{T} \mathbf{x}\right)=1$. Then $\mathbf{\Psi}^{T} \overline{\mathbf{A}}^{T} \mathbf{x}$ is a nonzero scalar multiple of one row of $\boldsymbol{\Psi}$. From (4.6), it follows that

$$
\begin{aligned}
\operatorname{rank}\left(\mathbf{B} \boldsymbol{\Phi} \operatorname{diag}\left(\Psi^{T} \mathbf{A}^{T} \mathbf{x}\right) \boldsymbol{\Omega}^{T} \mathbf{C}^{T}\right) & =\operatorname{rank}\left(\overline{\mathbf{B}} \boldsymbol{\Phi} \operatorname{diag}\left(\mathbf{\Psi}^{T} \overline{\mathbf{A}}^{T} \mathbf{x}\right) \boldsymbol{\Omega}^{T} \overline{\mathbf{C}}^{T}\right) \\
& \leq \operatorname{rank}\left(\boldsymbol{\Phi} \operatorname{diag}\left(\mathbf{\Psi}^{T} \overline{\mathbf{A}}^{T} \mathbf{x}\right) \boldsymbol{\Omega}^{T}\right) \\
& \leq \max _{j=1, \ldots, R_{1}}\left(\operatorname{rank}\left(\boldsymbol{\Phi} \operatorname{diag}\left(\boldsymbol{\psi}_{j}^{T}\right) \boldsymbol{\Omega}^{T}\right)\right) \\
& =N^{*} .
\end{aligned}
$$


Let $\mathbf{d}=\mathbf{A}^{T} \mathbf{x}$. If (4.7) implies $\omega(\mathbf{d}) \leq 1$, then the condition of the Permutation Lemma holds. The proof is complete by observing that this is exactly condition (4.4).

When PARALIND reduces to $\mathrm{CP}$, we have $N^{*}=1$ and $\boldsymbol{\Psi}=\boldsymbol{\Phi}=\boldsymbol{\Omega}=\mathbf{I}_{R}$, where we may have to permute the columns of $\mathbf{B}$ and $\mathbf{C}$ to obtain the latter identity (see Lemma 2.1). In this case, condition (4.4) is identical to Condition B of Jiang and Sidiropoulos [22] for the essential uniqueness of a CP component matrix of full column rank.

Condition (4.4) can be difficult to check. However, when $\mathbf{B}$ or $\mathbf{C}$ have full column rank, they can be eliminated from the expression $\operatorname{rank}\left(\mathbf{B} \boldsymbol{\Phi} \operatorname{diag}\left(\mathbf{\Psi}^{T} \mathbf{d}\right) \boldsymbol{\Omega}^{T} \mathbf{C}^{T}\right)$. If both $\mathbf{B}$ and $\mathbf{C}$ have full column rank, then condition (4.4) reduces to (4.8), which is easier to check. This is illustrated by the examples in Section 5. Also, $(\mathbf{B} \otimes \mathbf{C}) \mathbf{G}_{1}$ has full column rank if and only if $\mathbf{G}_{1}$ has full column rank. This yields the following corollary.

Corollary 4.3 Let $(\mathbf{A} \boldsymbol{\Psi}, \mathbf{B} \boldsymbol{\Phi}, \mathbf{C} \boldsymbol{\Omega})$ be a PARALIND solution for fixed constraint matrices $\mathbf{\Psi}, \mathbf{\Phi}$, and $\boldsymbol{\Omega}$. Let $(\overline{\mathbf{A}} \boldsymbol{\Psi}, \overline{\mathbf{B}} \boldsymbol{\Phi}, \overline{\mathbf{C}} \boldsymbol{\Omega})$ be an alternative PARALIND solution with the same residuals. Suppose $\mathbf{A}, \mathbf{B}, \mathbf{C}$ and $\mathbf{G}_{1}$ have full column rank. If for any vector $\mathbf{d}$,

$$
\operatorname{rank}\left(\boldsymbol{\Phi} \operatorname{diag}\left(\boldsymbol{\Psi}^{T} \mathbf{d}\right) \boldsymbol{\Omega}^{T}\right) \leq N^{*} \quad \text { implies } \quad \omega(\mathbf{d}) \leq 1,
$$

then there exists a unique permutation matrix $\boldsymbol{\Pi}$ and a unique nonsingular diagonal matrix $\boldsymbol{\Lambda}$ such that $\overline{\mathbf{A}}=\mathbf{A} \Pi \mathbf{\Lambda}$.

Note that when we use the Permutation Lemma to show that $\mathbf{S}$ in (4.2) is equal to $\mathbf{I}_{R_{1}}$ up to column scaling and permutation, then we obtain exactly condition (4.8) since $\mathbf{T}$ and $\mathbf{U}$ in (4.2) are nonsingular.

To check the uniqueness condition (4.8) requires solving a system of equations in the elements of the vector $\mathbf{d}$. In a special case, the following lemma states a condition that does not involve the vector $\mathbf{d}$.

Lemma 4.4 Let $\mathbf{A}, \mathbf{B}, \mathbf{C}$ and $\mathbf{G}_{1}$ have full column rank. If $\mathbf{\Psi}=\mathbf{I}_{R}$, then condition (4.8) holds if and only if $\mathbf{\Phi}$ and $\boldsymbol{\Omega}$ are $R \times R$ permutation matrices, i.e. if and only if we have the $C P$ model (see Lemma 2.1).

Proof. Let $\boldsymbol{\Psi}=\mathbf{I}_{R}$. It can be verified that $N^{*}=1$. Also, we have $\boldsymbol{\Phi} \operatorname{diag}\left(\boldsymbol{\Psi}^{T} \mathbf{d}\right) \boldsymbol{\Omega}^{T}=$ $\boldsymbol{\Phi} \operatorname{diag}(\mathbf{d}) \boldsymbol{\Omega}^{T}$. First, we show that condition (4.8) does not hold if $\boldsymbol{\Phi}$ and $\boldsymbol{\Omega}$ are not both $R \times R$ 
permutation matrices. Each element of $\mathbf{d}$ is contained in exactly one row of $\boldsymbol{\Phi} \operatorname{diag}(\mathbf{d})$ and some row of $\boldsymbol{\Phi} \operatorname{diag}(\mathbf{d})$ contains more than one $d_{j}$ if $\boldsymbol{\Phi}$ is not a permutation matrix. In that case, it is possible to set all $d_{j}$ equal to zero except those in a row containing multiple $d_{j}$. Then $\boldsymbol{\Phi} \operatorname{diag}(\mathbf{d})$ has rank 1 while $\omega(\mathbf{d}) \geq 2$. Hence, condition (4.8) does not hold. Analogously, it can be shown that if $\boldsymbol{\Omega}$ is not a permutation matrix, then there exists a $\mathbf{d} \operatorname{such}$ that $\operatorname{diag}(\mathbf{d}) \boldsymbol{\Omega}^{T}$ has rank 1 while $\omega(\mathbf{d}) \geq 2$.

It remains to show that condition (4.8) holds if $\boldsymbol{\Phi}$ and $\boldsymbol{\Omega}$ are $R \times R$ permutation matrices. But then $\operatorname{rank}\left(\boldsymbol{\Phi} \operatorname{diag}(\mathbf{d}) \boldsymbol{\Omega}^{T}\right)=\operatorname{rank}(\operatorname{diag}(\mathbf{d}))=\omega(\mathbf{d})$, which completes the proof.

\subsection{Necessary uniqueness conditions for CONFAC and PARALIND}

Additional to sufficient conditions for essential uniqueness in PARALIND, we also consider necessary uniqueness conditions. The lemma below follows from a necessary condition for CP essential uniqueness (see Stegeman and Sidiropoulos [38], p.543).

Lemma 4.5 Let $(\mathbf{A} \boldsymbol{\Psi}, \mathbf{B} \boldsymbol{\Phi}, \mathbf{C} \boldsymbol{\Omega})$ be a PARALIND solution for fixed constraint matrices $\boldsymbol{\Psi}$, $\boldsymbol{\Phi}$, and $\boldsymbol{\Omega}$. If $(\mathbf{B} \otimes \mathbf{C}) \mathbf{G}_{1}$ does not have full column rank, then $\mathbf{A}$ is not essentially unique. Moreover, an alternative PARALIND solution exists in which $\mathbf{A}$ has $R_{1}-1$ columns.

Proof. Suppose $(\mathbf{B} \otimes \mathbf{C}) \mathbf{G}_{1}$ does not have full column rank. Let $\mathbf{n}$ be such that $(\mathbf{B} \otimes \mathbf{C}) \mathbf{G}_{1} \mathbf{n}=\mathbf{0}$. Then

$$
(\mathbf{B} \otimes \mathbf{C}) \mathbf{G}_{1} \mathbf{A}^{T}=(\mathbf{B} \otimes \mathbf{C}) \mathbf{G}_{1}\left(\mathbf{A}+\mathbf{y} \mathbf{n}^{T}\right)^{T},
$$

for any vector $\mathbf{y}$. Hence, an alternative decomposition exists with $\left(\mathbf{A}+\mathbf{y n}^{T}\right)$ instead of $\mathbf{A}$. Moreover, we can choose $\mathbf{y}$ such that one column of $\left(\mathbf{A}+\mathbf{y} \mathbf{n}^{T}\right)$ becomes all-zero. This completes the proof.

Another necessary condition for CP essential uniqueness is that none of the component matrices may have all-zero or proportional columns (see Stegeman and Sidiropoulos [38], p.543). For CONFAC we have a similar condition: if, for some $(s, t)$, columns $s$ and $t$ of $\boldsymbol{\Psi}$ are identical and columns $s$ and $t$ are unique in $\boldsymbol{\Phi}$ and $\boldsymbol{\Omega}$, then $\mathbf{B}$ and $\mathbf{C}$ are not unique. In the lemma below, a more general form of this condition is proven.

Lemma 4.6 Let $(\mathbf{A} \boldsymbol{\Psi}, \mathbf{B} \boldsymbol{\Phi}, \mathbf{C} \boldsymbol{\Omega})$ be a CONFAC solution for fixed constraint matrices $\mathbf{\Psi}$, $\mathbf{\Phi}$, and $\boldsymbol{\Omega}$. Suppose, for some column index set $\mathcal{S} \subseteq\{1, \ldots, R\}$ with at least two elements, columns $\mathcal{S}$ of 
$\boldsymbol{\Psi}$ are identical and columns $\mathcal{S}$ of $\boldsymbol{\Phi}$ and $\boldsymbol{\Omega}$ do not repeat in columns $\{1, \ldots, R\} \backslash \mathcal{S}$ of $\boldsymbol{\Phi}$ and $\boldsymbol{\Omega}$. If columns $\mathcal{S}$ of $\mathbf{\Phi}$ and $\boldsymbol{\Omega}$ are not all identical, then $\mathbf{B}$ and $\mathbf{C}$ are not essentially unique.

Proof. For simplicity, let columns $\mathcal{S}$ of $\boldsymbol{\Psi}$ be equal to the first column of $\mathbf{I}_{R_{1}}$. We denote the columns $\mathcal{S}$ of $\boldsymbol{\Phi}$ and $\boldsymbol{\Omega}$ by $\boldsymbol{\Phi}_{\mathcal{S}}$ and $\boldsymbol{\Omega}_{\mathcal{S}}$, respectively. Let the unique columns of $\mathbf{B}$ and $\mathbf{C}$ in $\mathbf{B} \boldsymbol{\Phi}_{\mathcal{S}}$ and $\mathbf{C} \boldsymbol{\Omega}_{\mathcal{S}}$ be given by $\mathbf{B}_{\mathcal{S}}\left(J \times n_{2}\right)$ and $\mathbf{C}_{\mathcal{S}}\left(K \times n_{3}\right)$, respectively. Then the CONFAC decomposition is

$$
\mathbf{a}_{1} \circ \mathbf{B} \boldsymbol{\Phi}_{\mathcal{S}}\left(\mathbf{C} \boldsymbol{\Omega}_{\mathcal{S}}\right)^{T}+(\text { rest })
$$

where (rest) does not include the columns $\mathbf{B}_{\mathcal{S}}$ and $\mathbf{C}_{\mathcal{S}}$ of $\mathbf{B}$ and $\mathbf{C}$, respectively. The number of rank-1 terms in the first part of $(4.10)$ equals $\operatorname{card}(\mathcal{S})$. We have

$$
\mathbf{B} \Phi_{\mathcal{S}}\left(\mathbf{C} \boldsymbol{\Omega}_{\mathcal{S}}\right)^{T}=\mathbf{B}_{\mathcal{S}} \mathbf{V} \mathbf{C}_{\mathcal{S}}^{T}
$$

with $\mathbf{V}$ being an $n_{2} \times n_{3}$ matrix. Let $m=\min \left(n_{2}, n_{3}\right)$. The condition of the lemma implies that $\max \left(n_{2}, n_{3}\right) \geq 2$. For any matrices $\mathbf{T}\left(n_{2} \times m\right)$ and $\mathbf{U}\left(n_{3} \times m\right)$ with $\mathbf{V}=\mathbf{T U}^{T}$, an alternative decomposition is

$$
\mathbf{a}_{1} \circ \mathbf{B}_{\mathcal{S}} \mathbf{T}\left(\mathbf{C}_{\mathcal{S}} \mathbf{U}\right)^{T}+(\text { rest })
$$

The number of rank-1 terms in the first part of (4.12) equals $m$. Hence, we have shown that $\mathbf{B}$ and C are not essentially unique. This completes the proof.

Note that, in order to obtain conditions for essential uniqueness of $\mathbf{B}$ and $\mathbf{C}$, it suffices to interchange the roles of $(\mathbf{A}, \boldsymbol{\Psi}),(\mathbf{B}, \mathbf{\Phi})$ and $(\mathbf{C}, \boldsymbol{\Omega})$ in Theorem 4.2, Corollary 4.3, and Lemmas 4.5 and 4.6.

\section{$5 \quad$ Examples}

In this section, we present two examples to demonstrate the condition (4.8) of Corollary 4.3 for essential uniqueness. In all examples it is implicitly assumed that $\mathbf{A}, \mathbf{B}$, and $\mathbf{C}$ have full column rank. The first example is Example 2 in de Almeida et al. [3]. Here, we have a CONFAC model with $R=4, R_{1}=R_{2}=3, R_{3}=2$, and

$$
\boldsymbol{\Psi}=\left[\begin{array}{llll}
1 & 1 & 0 & 0 \\
0 & 0 & 0 & 1 \\
0 & 0 & 1 & 0
\end{array}\right], \quad \boldsymbol{\Phi}=\left[\begin{array}{llll}
0 & 0 & 1 & 0 \\
1 & 1 & 0 & 0 \\
0 & 0 & 0 & 1
\end{array}\right], \quad \boldsymbol{\Omega}=\left[\begin{array}{llll}
1 & 1 & 1 & 0 \\
0 & 0 & 0 & 1
\end{array}\right]
$$


Using our results in Section 4, we show that $\mathbf{C}$ is essentially unique. It can be verified that $\mathbf{G}_{3}$ has full column rank. We have

$$
\boldsymbol{\Phi} \operatorname{diag}\left(\boldsymbol{\omega}_{1}^{T}\right) \boldsymbol{\Psi}^{T}=\left[\begin{array}{ccc}
0 & 0 & 1 \\
2 & 0 & 0 \\
0 & 0 & 0
\end{array}\right], \quad \boldsymbol{\Phi} \operatorname{diag}\left(\boldsymbol{\omega}_{2}^{T}\right) \boldsymbol{\Psi}^{T}=\left[\begin{array}{ccc}
0 & 0 & 0 \\
0 & 0 & 0 \\
0 & 1 & 0
\end{array}\right]
$$

where $\omega_{j}^{T}$ denotes row $j$ of $\boldsymbol{\Omega}$. Hence, $N^{*}=2$ follows from its definition (4.3) with $\boldsymbol{\Psi}$ and $\boldsymbol{\Omega}$ interchanged. Next, we check condition (4.8) translated to C. For a vector $\mathbf{d}=\left(d_{1} d_{2}\right)^{T}$, we obtain

$$
\boldsymbol{\Phi} \operatorname{diag}\left(\boldsymbol{\Omega}^{T} \mathbf{d}\right) \boldsymbol{\Psi}^{T}=\left[\begin{array}{ccc}
0 & 0 & d_{1} \\
2 d_{1} & 0 & 0 \\
0 & d_{2} & 0
\end{array}\right]
$$

If the matrix in (5.3) is to have rank at most 2 , it follows that $d_{1} d_{2}=0$. Hence, the condition (4.8) translated to $\mathbf{C}$ holds and $\mathbf{C}$ is essentially unique.

It follows from (5.1) that the CONFAC decomposition is given by

$$
\mathbf{a}_{1} \circ \mathbf{b}_{2} \circ \mathbf{c}_{1}+\mathbf{a}_{1} \circ \mathbf{b}_{2} \circ \mathbf{c}_{1}+\mathbf{a}_{3} \circ \mathbf{b}_{1} \circ \mathbf{c}_{1}+\mathbf{a}_{2} \circ \mathbf{b}_{3} \circ \mathbf{c}_{2}
$$

An alternative decomposition is

$$
\left(\mathbf{a}_{1}+\mathbf{a}_{3}\right) \circ \mathbf{b}_{1} \circ \mathbf{c}_{1}+\mathbf{a}_{1} \circ\left(2 \mathbf{b}_{2}-\mathbf{b}_{1}\right) \circ \mathbf{c}_{1}+\mathbf{a}_{2} \circ \mathbf{b}_{3} \circ \mathbf{c}_{2} .
$$

This shows that $\mathbf{A}$ and $\mathbf{B}$ are not essentially unique.

The second example is taken from Bro et al. [6, section 3.2.5]. Here, we have a PARALIND model with $R=6, R_{1}=3, R_{2}=6, R_{3}=4, \mathbf{\Phi}=\mathbf{I}_{6}$ and

$$
\boldsymbol{\Psi}=\left[\begin{array}{cccccc}
1 & 1 & 0 & 0 & 0 & 0 \\
0 & 0 & 1 & 1 & 0 & 0 \\
0 & 0 & 0 & 0 & 1 & 1
\end{array}\right], \quad \boldsymbol{\Omega}=\left[\begin{array}{cccccc}
1 & -1 & 0 & 0 & 0 & 0 \\
0 & 0 & 1 & -1 & 0 & 0 \\
0 & 0 & 0 & 0 & 1 & -1 \\
0 & 1 & 0 & 1 & 0 & 1
\end{array}\right] \text {. }
$$

Using Corollary 4.3, we show that $\mathbf{A}$ is essentially unique. It can be verified that $\mathbf{G}_{1}$ has full column 
rank and that $N^{*}=2$. Next, we check condition (4.8). For a vector $\mathbf{d}=\left(d_{1} d_{2} d_{3}\right)^{T}$, we obtain

$$
\boldsymbol{\Phi} \operatorname{diag}\left(\mathbf{\Psi}^{T} \mathbf{d}\right) \boldsymbol{\Omega}^{T}=\left[\begin{array}{cccc}
d_{1} & 0 & 0 & 0 \\
-d_{1} & 0 & 0 & d_{1} \\
0 & d_{2} & 0 & 0 \\
0 & -d_{2} & 0 & d_{2} \\
0 & 0 & d_{3} & 0 \\
0 & 0 & -d_{3} & d_{3}
\end{array}\right] .
$$

If the matrix in (5.7) is to have rank at most 2 , it follows that $\omega(\mathbf{d}) \leq 1$. Hence, condition (4.8) holds and $\mathbf{A}$ is essentially unique. This is not proven in [6].

\section{A partial uniqueness condition for PARALIND}

Here, we present a partial uniqueness condition for one component matrix in PARALIND. Without loss of generality, we focus on the partial uniqueness of $\mathbf{A}$. We obtain our partial uniqueness condition by applying the approach of Jiang and Sidiropoulos [22] to the PARALIND decomposition in which the columns of $\mathbf{A}$ are partitioned into disjoint subsets. The structure of our proof is analogous to the proof of the essential uniqueness condition in Theorem 4.2. Instead of Kruskal's Permutation Lemma [26], we make use of the Equivalence Lemma for Partitioned Matrices that was proven by De Lathauwer [11]. This lemma is used by De Lathauwer [12] to prove partial uniqueness results of the partitioned component matrices of the decomposition in $\operatorname{rank}-(L, M, N)$ terms. The Equivalence Lemma for Partitioned Matrices is formulated as follows. For a vector y partitioned as $\mathbf{y}=\left(\mathbf{y}_{1}^{T}|\ldots| \mathbf{y}_{F}^{T}\right)^{T}$, let $\omega^{\prime}(\mathbf{y})$ denote the number of parts of the vector that are not all-zero. For a matrix $\mathbf{A}$ partitioned as $\mathbf{A}=\left[\mathbf{A}_{1}|\ldots| \mathbf{A}_{F}\right]$, let $k_{\mathbf{A}}^{\prime}$ denote the maximal number $f$ such that any set of $f$ submatrices of $\mathbf{A}$ has full column rank. Note that this generalizes the concept of k-rank to partitioned matrices.

Lemma 6.1 (Equivalence Lemma for Partitioned Matrices) Let $\mathbf{A}$ and $\overline{\mathbf{A}}$ be two $I \times R_{1}$ matrices partitioned in the same way into F submatrices that are of full column rank. Suppose the following condition holds: for any vector $\mathbf{x}$ such that $\omega^{\prime}\left(\overline{\mathbf{A}}^{T} \mathbf{x}\right) \leq F-k_{\overline{\mathbf{A}}}^{\prime}+1$, we have $\omega^{\prime}\left(\mathbf{A}^{T} \mathbf{x}\right) \leq \omega^{\prime}\left(\overline{\mathbf{A}}^{T} \mathbf{x}\right)$. Then there exists a unique block-permutation matrix $\boldsymbol{\Pi}$ and a unique nonsingular block-diagonal matrix $\boldsymbol{\Lambda}$ such that $\overline{\mathbf{A}}=\mathbf{A} \boldsymbol{\Pi} \boldsymbol{\Lambda}$, where the block-transformation is compatible with the partition of $\mathbf{A}$ and $\overline{\mathbf{A}}$. 
Note that Lemma 6.1 is not a straightforward generalization of the Permutation Lemma (Lemma 4.1). The condition of Lemma 4.1 features $\omega\left(\overline{\mathbf{A}}^{T} \mathbf{x}\right) \leq R_{1}-\operatorname{rank}(\overline{\mathbf{A}})+1$, while in Lemma 6.1 this becomes $\omega^{\prime}\left(\overline{\mathbf{A}}^{T} \mathbf{x}\right) \leq F-k_{\overline{\mathbf{A}}}^{\prime}+1$. Hence, the rank of $\overline{\mathbf{A}}$ has been replaced by the $\mathrm{k}^{\prime}$-rank of $\overline{\mathbf{A}}$. It can be shown by an example that Lemma 6.1 with an $\mathrm{r}^{\prime}$-rank of $\overline{\mathbf{A}}$ is incorrect.

Our partial uniqueness condition for PARALIND is the following.

Theorem 6.2 Let $(\mathbf{A} \boldsymbol{\Psi}, \mathbf{B} \mathbf{\Phi}, \mathbf{C} \boldsymbol{\Omega})$ be a PARALIND solution for fixed constraint matrices $\mathbf{\Psi}, \mathbf{\Phi}$, and $\boldsymbol{\Omega}$. Let $(\overline{\mathbf{A}} \boldsymbol{\Psi}, \overline{\mathbf{B}} \boldsymbol{\Phi}, \overline{\mathbf{C}} \boldsymbol{\Omega})$ be an alternative PARALIND solution with the same residuals. Let the columns of $\mathbf{A}$ be partitioned as $\mathbf{A} \boldsymbol{\Pi}_{a}=\left[\mathbf{A}_{1}|\ldots| \mathbf{A}_{F}\right]$, where $\boldsymbol{\Pi}_{a}$ is a permutation matrix. Define the partition of $\overline{\mathbf{A}}$ analogously as $\overline{\mathbf{A}} \boldsymbol{\Pi}_{a}=\left[\overline{\mathbf{A}}_{1}|\ldots| \overline{\mathbf{A}}_{F}\right]$. Let

$$
N^{* *}=\max _{f=1, \ldots, F} \max _{\mathbf{g}_{f}}\left(\operatorname{rank}\left(\boldsymbol{\Phi} \operatorname{diag}\left(\mathbf{\Psi}^{T} \mathbf{\Pi}_{a} \mathbf{g}_{f}\right) \boldsymbol{\Omega}^{T}\right)\right)
$$

where $\mathbf{g}_{f}$ is a vector with the same partition as $\mathbf{A} \mathbf{\Pi}_{a}$ and nonzero elements only in part $f$. Suppose $(\mathbf{B} \otimes \mathbf{C}) \mathbf{G}_{1}$ and $\mathbf{A}$ have full column rank. If for any vector $\mathbf{d}$ with the same partition as $\mathbf{A} \mathbf{\Pi}_{a}$,

$$
\operatorname{rank}\left(\mathbf{B} \boldsymbol{\Phi} \operatorname{diag}\left(\boldsymbol{\Psi}^{T} \boldsymbol{\Pi}_{a} \mathbf{d}\right) \boldsymbol{\Omega}^{T} \mathbf{C}^{T}\right) \leq N^{* *} \quad \text { implies } \quad \omega^{\prime}(\mathbf{d}) \leq 1,
$$

then there exists a unique block-permutation matrix $\boldsymbol{\Pi}$ and a unique nonsingular block-diagonal matrix $\boldsymbol{\Lambda}$ such that $\overline{\mathbf{A}} \boldsymbol{\Pi}_{a}=\mathbf{A} \boldsymbol{\Pi}_{a} \boldsymbol{\Pi} \boldsymbol{\Lambda}$, where the block-transformation is compatible with the partition of $\mathbf{A} \boldsymbol{\Pi}_{a}$ and $\overline{\mathbf{A}} \boldsymbol{\Pi}_{a}$.

Proof. See Appendix.

The result of Theorem 6.2 states that each subset of columns of $\overline{\mathbf{A}} \boldsymbol{\Pi}_{a}$ satisfies $\overline{\mathbf{A}}_{f}=\mathbf{A}_{\pi(f)} \mathbf{S}$, where $\mathbf{S}$ is a unique nonsingular matrix and the permutation $\pi(\cdot)$ is defined by the unique blockpermutation $\boldsymbol{\Pi}$. Hence, according to Definition 3.2, $\mathbf{A}$ is partially unique. Note that when checking the condition of Theorem 6.2 , one is free to choose a suitable column permutation $\boldsymbol{\Pi}_{a}$ and partition of $\mathbf{A} \boldsymbol{\Pi}_{a}$.

Theorem 6.2 is a generalization of Theorem 4.2. Indeed, if the column permutation $\boldsymbol{\Pi}_{a}$ equals $\mathbf{I}_{R}$ and the disjoint subsets in the partition of the columns of $\mathbf{A}$ are the columns themselves, then Theorem 6.2 is just Theorem 4.2 .

Condition (6.2) can be difficult to check. However, when $\mathbf{B}$ or $\mathbf{C}$ have full column rank, they can be eliminated from the expression $\operatorname{rank}\left(\mathbf{B} \boldsymbol{\Phi} \operatorname{diag}\left(\boldsymbol{\Psi}^{T} \boldsymbol{\Pi}_{a} \mathbf{d}\right) \boldsymbol{\Omega}^{T} \mathbf{C}^{T}\right)$. If both $\mathbf{B}$ and $\mathbf{C}$ have full column rank, then condition (6.2) reduces to (6.3) which is easier to check. This is illustrated by 
the examples in Section 7. Also, $(\mathbf{B} \otimes \mathbf{C}) \mathbf{G}_{1}$ has full column rank if and only if $\mathbf{G}_{1}$ has full column rank. This yields the following analogue of Corollary 4.3.

Corollary 6.3 Let $(\mathbf{A} \boldsymbol{\Psi}, \mathbf{B} \boldsymbol{\Phi}, \mathbf{C} \boldsymbol{\Omega})$ be a PARALIND solution for fixed constraint matrices $\mathbf{\Psi}, \mathbf{\Phi}$, and $\boldsymbol{\Omega}$. Let $(\overline{\mathbf{A}} \boldsymbol{\Psi}, \overline{\mathbf{B}} \boldsymbol{\Phi}, \overline{\mathbf{C}} \boldsymbol{\Omega})$ be an alternative PARALIND solution with the same residuals. Let the columns of $\mathbf{A}$ be partitioned as $\mathbf{A} \boldsymbol{\Pi}_{a}=\left[\mathbf{A}_{1}|\ldots| \mathbf{A}_{F}\right]$, where $\boldsymbol{\Pi}_{a}$ is a permutation matrix. Define the partition of $\overline{\mathbf{A}}$ analogously as $\overline{\mathbf{A}} \boldsymbol{\Pi}_{a}=\left[\overline{\mathbf{A}}_{1}|\ldots| \overline{\mathbf{A}}_{F}\right]$. Suppose $\mathbf{A}, \mathbf{B}, \mathbf{C}$ and $\mathbf{G}_{1}$ have full column rank. If for any vector $\mathbf{d}$ with the same partition as $\mathbf{A} \boldsymbol{\Pi}_{a}$,

$$
\operatorname{rank}\left(\boldsymbol{\Phi} \operatorname{diag}\left(\boldsymbol{\Psi}^{T} \boldsymbol{\Pi}_{a} \mathbf{d}\right) \boldsymbol{\Omega}^{T}\right) \leq N^{* *} \quad \text { implies } \quad \omega^{\prime}(\mathbf{d}) \leq 1
$$

then there exists a unique block-permutation matrix $\boldsymbol{\Pi}$ and a unique nonsingular block-diagonal matrix $\boldsymbol{\Lambda}$ such that $\overline{\mathbf{A}} \boldsymbol{\Pi}_{a}=\mathbf{A} \boldsymbol{\Pi}_{a} \boldsymbol{\Pi} \boldsymbol{\Lambda}$, where the block-transformation is compatible with the partition of $\mathbf{A} \boldsymbol{\Pi}_{a}$ and $\overline{\mathbf{A}} \boldsymbol{\Pi}_{a}$.

Note that, in order to obtain conditions for partial uniqueness of subsets of columns of $\mathbf{B}$ and $\mathbf{C}$, it suffices to interchange the roles of $(\mathbf{A}, \mathbf{\Psi}),(\mathbf{B}, \mathbf{\Phi})$ and $(\mathbf{C}, \boldsymbol{\Omega})$ in Theorem 6.2 and Corollary 6.3.

\section{$7 \quad$ Examples}

In this section, we present two examples to demonstrate the condition (6.3) of Corollary 6.3 for partial uniqueness. In the examples it is implicitly assumed that $\mathbf{A}, \mathbf{B}$, and $\mathbf{C}$ have full column rank.

As a first example, consider again the CONFAC model with constraint matrices (5.1). In Section 5 it was established that $\mathbf{C}$ is essentially unique, while $\mathbf{A}$ and $\mathbf{B}$ are not essentially unique. Next, we show that the subsets of columns in $\mathbf{A} \boldsymbol{\Pi}_{a}=\left[\mathbf{a}_{1} \mathbf{a}_{3} \mid \mathbf{a}_{2}\right]$ are partially unique. It can be verified that $\mathbf{G}_{1}$ has full column rank. We have

$$
\boldsymbol{\Psi}^{T} \boldsymbol{\Pi}_{a}=\left[\begin{array}{ccc}
1 & 0 & 0 \\
1 & 0 & 0 \\
0 & 1 & 0 \\
0 & 0 & 1
\end{array}\right] .
$$


For $\mathbf{g}_{1}=\left(\begin{array}{lll}\beta \gamma & 0\end{array}\right)^{T}$ and $\mathbf{g}_{2}=\left(\begin{array}{lll}0 & 0 & \alpha\end{array}\right)^{T}$, we obtain

$$
\boldsymbol{\Phi} \operatorname{diag}\left(\boldsymbol{\Psi}^{T} \boldsymbol{\Pi}_{a} \mathbf{g}_{1}\right) \boldsymbol{\Omega}^{T}=\left[\begin{array}{cc}
\gamma & 0 \\
2 \beta & 0 \\
0 & 0
\end{array}\right] \quad \boldsymbol{\Phi} \operatorname{diag}\left(\boldsymbol{\Psi}^{T} \boldsymbol{\Pi}_{a} \mathbf{g}_{2}\right) \boldsymbol{\Omega}^{T}=\left[\begin{array}{cc}
0 & 0 \\
0 & 0 \\
0 & \alpha
\end{array}\right]
$$

where $\alpha, \beta$ and $\gamma$ are arbitrary nonzero numbers. This implies $N^{* *}=1$, see (6.1). Next, we check whether condition (6.3) holds. For a vector $\mathbf{d}$ partitioned as $\mathbf{d}=\left(d_{1} d_{2} \mid d_{3}\right)^{T}$ we have

$$
\boldsymbol{\Phi} \operatorname{diag}\left(\boldsymbol{\Psi}^{T} \boldsymbol{\Pi}_{a} \mathbf{d}\right) \boldsymbol{\Omega}^{T}=\left[\begin{array}{cc}
d_{2} & 0 \\
2 d_{1} & 0 \\
0 & d_{3}
\end{array}\right] .
$$

If this matrix is to have rank at most 1 , it follows that $d_{1} d_{3}=d_{2} d_{3}=0$. Hence, $\omega^{\prime}(\mathbf{d}) \leq 1$ holds and the two subsets of columns in $\mathbf{A} \boldsymbol{\Pi}_{a}$ are partially unique. Note that this implies that $\mathbf{a}_{2}$ is unique up to scaling, $\mathbf{a}_{1}$ and $\mathbf{a}_{3}$ are identified up to their linear span, and the two subsets of columns may appear in a different order in $\overline{\mathbf{A}} \boldsymbol{\Pi}_{a}$. By translating condition (6.3) to $\mathbf{B}$, it follows that $\mathbf{B} \boldsymbol{\Pi}_{b}=\left[\mathbf{b}_{1} \mathbf{b}_{2} \mid \mathbf{b}_{3}\right]$ is also partially unique.

The second example is taken from Bro et al. [6, section 3.2.4]. Here, we have a CONFAC model with $R=6, R_{1}=3, R_{2}=R_{3}=6$, and

$$
\boldsymbol{\Psi}=\left[\begin{array}{llllll}
1 & 1 & 0 & 0 & 0 & 0 \\
0 & 0 & 1 & 1 & 0 & 0 \\
0 & 0 & 0 & 0 & 1 & 1
\end{array}\right], \quad \boldsymbol{\Phi}=\boldsymbol{\Omega}=\mathbf{I}_{6}
$$

Using Corollary 4.3, it can be verified that $\mathbf{A}$ is essentially unique. Lemma 4.6 with $\mathcal{S}=\{1,2\}$ yields that $\mathbf{B}$ and $\mathbf{C}$ are not essentially unique. Next, we show that $\mathbf{B}=\left[\mathbf{b}_{1} \mathbf{b}_{2}\left|\mathbf{b}_{3} \mathbf{b}_{4}\right| \mathbf{b}_{5} \mathbf{b}_{6}\right]$ is partially unique. Since $\mathbf{\Phi}=\boldsymbol{\Omega}$ it immediately follows that $\mathbf{C}=\left[\mathbf{c}_{1} \mathbf{c}_{2}\left|\mathbf{c}_{3} \mathbf{c}_{4}\right| \mathbf{c}_{5} \mathbf{c}_{6}\right]$ is also partially unique. It can be verified that $\mathbf{G}_{2}$ has full column rank. Interchanging $\boldsymbol{\Psi}$ and $\mathbf{\Phi}$ in the definition of $N^{* *}$ in (6.1), we obtain $N^{* *}=1$. Interchanging $\boldsymbol{\Psi}$ and $\boldsymbol{\Phi}$ in condition (6.3) yields

$$
\boldsymbol{\Psi} \operatorname{diag}\left(\boldsymbol{\Phi}^{T} \mathbf{d}\right) \boldsymbol{\Omega}^{T}=\left[\begin{array}{cccccc}
d_{1} & d_{2} & 0 & 0 & 0 & 0 \\
0 & 0 & d_{3} & d_{4} & 0 & 0 \\
0 & 0 & 0 & 0 & d_{5} & d_{6}
\end{array}\right] .
$$

If this matrix is to have rank at most 1 , it follows that at least two of the pairs $\left(d_{1}, d_{2}\right),\left(d_{3}, d_{4}\right)$,

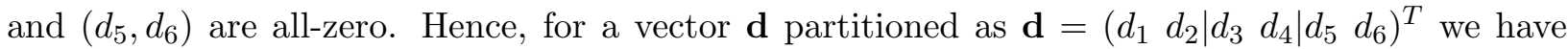

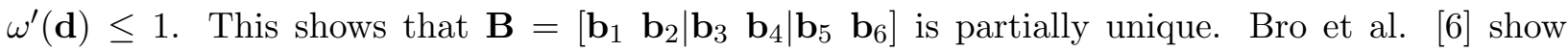


the essential uniqueness of $\mathbf{A}$ and partial uniqueness of $\mathbf{B}$ and $\mathbf{C}$ using an eigendecomposition argument.

Note that our partial uniqueness condition for PARALIND can also be applied to CP in case one of the component matrices has proportional columns. Indeed, let $\mathbf{A}(I \times R-1), \mathbf{B}(J \times R)$ and $\mathbf{C}(K \times R)$ have full column rank, $\mathbf{\Phi}=\boldsymbol{\Omega}=\mathbf{I}_{R}$, and $\mathbf{\Psi}=\left[\alpha \mathbf{e}_{1} \mid \mathbf{I}_{R-1}\right](R-1 \times R)$, with $\alpha \neq 0$. As in the last example, we can show that the first two columns of $\mathbf{B}$ and $\mathbf{C}$ are identified up to their linear span only. This approach can be extended to the case where $\mathbf{A} \boldsymbol{\Psi}$ has several groups of proportional columns as in the example above.

\section{Uniqueness for constrained Tucker3 models}

As explained at the beginning of Section 4, studying PARALIND uniqueness is analogous to studying uniqueness of Tucker3 models with a constrained core array (i.e. containing many zero elements). Here, we discuss how the results in this paper may benefit the study of uniqueness of constrained Tucker3 models, and how the latter benefits the study of PARALIND uniqueness. Uniqueness results for Tucker3 models with a constrained core array have been obtained by a.o. Kiers et al. [23] (for a family of constrained $3 \times 3 \times 3$ cores), and Ten Berge and Smilde [41] (for a particular constrained $5 \times 3 \times 3$ core). See also Ten Berge [43] and the references therein. Ten Berge and Smilde [41] express the need for systematic study of uniqueness in constrained Tucker3 models, since current results resort to ad hoc arguments. This is exactly where the uniqueness conditions in Sections 4 and 6 may be useful. They represent a systematic analysis of uniqueness that can handle not only essential uniqueness but also partial uniqueness as defined in Definition 3.2. Any Tucker3 model with a core array with several zero elements can be written as a PARALIND model, and our PARALIND uniqueness conditions can be applied.

Conversely, the study of PARALIND uniqueness also benefits from the study of uniqueness of constrained Tucker3 models. As an example, we consider the following uniqueness result from Kiers et al. [23].

Proposition 8.1 Let $(\mathbf{A}, \mathbf{B}, \mathbf{C})$ be a Tucker3 solution with $3 \times 3 \times 3$ core array $\mathbf{G}$ with slices

$$
\left[\begin{array}{lll}
1 & 0 & 0 \\
0 & 0 & b \\
0 & a & 0
\end{array}\right], \quad\left[\begin{array}{lll}
0 & 0 & d \\
0 & 1 & 0 \\
c & 0 & 0
\end{array}\right], \quad\left[\begin{array}{lll}
0 & f & 0 \\
e & 0 & 0 \\
0 & 0 & 1
\end{array}\right] .
$$


If $\mathbf{A}, \mathbf{B}$, and $\mathbf{C}$ have full column rank and $(1+a d e+b c f)^{3} \neq 27 a b c d e f$, then $\mathbf{A}, \mathbf{B}$, and $\mathbf{C}$ are essentially unique.

The case $a=b=c=d=e=0$ and $f=1$ corresponds to a CONFAC model with

$$
\boldsymbol{\Psi}=\left[\begin{array}{llll}
1 & 0 & 0 & 1 \\
0 & 1 & 0 & 0 \\
0 & 0 & 1 & 0
\end{array}\right], \quad \boldsymbol{\Phi}=\left[\begin{array}{llll}
1 & 0 & 0 & 0 \\
0 & 1 & 0 & 1 \\
0 & 0 & 1 & 0
\end{array}\right], \quad \boldsymbol{\Omega}=\left[\begin{array}{llll}
1 & 0 & 0 & 0 \\
0 & 1 & 0 & 0 \\
0 & 0 & 1 & 1
\end{array}\right]
$$

It can be verified that the condition (4.8) for essential uniqueness does not hold for $\mathbf{A}$ or $\mathbf{B}$ or C. However, from Proposition 8.1 we know that all component matrices are essentially unique. Analogously, the essential uniqueness of other CONFAC or PARALIND models with a core array (2.4) of the form (8.1) can be obtained.

\section{Uniqueness for the block decomposition in rank- $(L, L, 1)$ terms}

Here, we discuss uniqueness in the case where CONFAC is equal to the block decomposition in rank- $(L, L, 1)$ terms, introduced by De Lathauwer [12]. As explained in Section 2, we have $\mathbf{A}=$ $\left[\mathbf{A}_{1}|\ldots| \mathbf{A}_{F}\right], \mathbf{B}=\left[\mathbf{B}_{1}|\ldots| \mathbf{B}_{F}\right], \mathbf{C}=\left[\mathbf{c}_{1} \ldots \mathbf{c}_{F}\right], \mathbf{\Psi}=\mathbf{\Phi}=\mathbf{I}_{L F}$, and $\boldsymbol{\Omega}=\mathbf{I}_{F} \otimes \mathbf{1}_{L}^{T}$. Here, $\mathbf{A}_{f}$ and $\mathbf{B}_{f}$ are $I \times L$ and $J \times L$, respectively, and have full column rank for all $f$. First we show, analogous to the approach of Jiang and Sidiropoulos [22] for CP, that if $\mathbf{C}$ has full column rank, then essential uniqueness of $\mathbf{C}$ implies partial uniqueness of $\mathbf{A}$ and B. Next, also analogous to [22], we present a necessary and sufficient condition for essential uniqueness of $\mathbf{C}$. We show that this condition is identical to condition (4.4) in Theorem 4.2 (translated to $\mathbf{C}$ ).

Proposition 9.1 Let $(\mathbf{A}, \mathbf{B}, \mathbf{C})$ be a solution for the block decomposition in rank- $(L, L, 1)$ terms, with $\mathbf{C}$ having full column rank. Suppose $\mathbf{C}$ is essentially unique as defined in Definition 3.1. Then $\mathbf{A}$ and $\mathbf{B}$ are partially unique as defined in Definition 3.2, i.e. the parts $\mathbf{A}_{f}$ and $\mathbf{B}_{f}$ are identified up to their column spaces.

Proof. See Appendix.

As in Jiang and Sidiropoulos [22] we obtain the following necessary and sufficient condition for the uniqueness of $(\mathbf{A}, \mathbf{B}, \mathbf{C})$. 
Proposition 9.2 Let $(\mathbf{A}, \mathbf{B}, \mathbf{C})$ be a solution for the block decomposition in rank- $(L, L, 1)$ terms, with $\mathbf{C}$ having full column rank. Then $\mathbf{A}$ and $\mathbf{B}$ are partially unique and $\mathbf{C}$ is essentially unique if and only if

$$
\operatorname{rank}\left(d_{1} \mathbf{A}_{1} \mathbf{B}_{1}^{T}+\cdots+d_{F} \mathbf{A}_{F} \mathbf{B}_{F}^{T}\right) \leq L \quad \text { implies } \quad \omega\left(d_{1}, \ldots, d_{F}\right) \leq 1 .
$$

Proof. See Appendix.

The results in this section have been obtained by following the approach of Jiang and Sidiropoulos [22]. Since that same approach is used to derive Theorem 4.2, it may not come as a surprise that condition (9.1) is the same as condition (4.4) translated to $\mathbf{C}$. Indeed, interchanging the roles of $(\mathbf{A}, \mathbf{\Psi})$ and $(\mathbf{C}, \boldsymbol{\Omega})$ we obtain $N^{*}=\max _{j}\left(\operatorname{rank}\left(\operatorname{diag}\left(\boldsymbol{\omega}_{j}^{T}\right)\right)=L\right.$ and

$$
\operatorname{rank}\left(\mathbf{B} \operatorname{diag}\left(\boldsymbol{\Omega}^{T} \mathbf{d}\right) \mathbf{A}^{T}\right)=\operatorname{rank}\left(d_{1} \mathbf{A}_{1} \mathbf{B}_{1}^{T}+\cdots+d_{F} \mathbf{A}_{F} \mathbf{B}_{F}^{T}\right) .
$$

For CP, Jiang and Sidiropoulos [22] show that their difficult-to-check necessary and sufficient condition for uniqueness (this is (9.1) with $L=1$ ) is implied by the condition that $\mathbf{U}_{(\mathbf{A}, \mathbf{B})}$ has full column rank, where $\mathbf{U}_{(\mathbf{A}, \mathbf{B})}$ is a matrix containing products of $2 \times 2$ minors of $\mathbf{A}$ and $\mathbf{B}$. This condition is much easier to check in practice. An alternative proof of this condition is given by De Lathauwer [10], who uses a link between $\mathrm{CP}$ and the problem of simultaneous matrix diagonalization. Nion and De Lathauwer [27] present a generalization of [10] to the block decomposition in rank- $(L, L, 1)$ terms.

Using an eigendecomposition argument, Sidiropoulos and Dimić [30] prove essential uniqueness of $\mathbf{C}$ and partial uniqueness of the partitioned matrices $\mathbf{A}$ and $\mathbf{B}$ for a case where $\mathbf{C}$ does not have full column rank. Here, we cannot use Proposition 9.2. However, using Theorem 4.2 for $\mathbf{C}$ and Theorem 6.2 for $\mathbf{A}$ and $\mathbf{B}$ yields the same uniqueness properties as in [30].

\section{Discussion}

In this paper, we considered the PARALIND model which is a restricted $\mathrm{CP}$ model in which the vectors forming the outer product arrays are linearly dependent according to a prespecified pattern. An important special case of PARALIND is CONFAC, in which the linear dependencies take the form of identical columns. PARALIND and CONFAC can also be considered as Tucker3 models with a constrained core array. Moreover, some types of block decompositions are special cases of CONFAC or PARALIND. Using the approach of Jiang and Sidiropoulos [22] for CP uniqueness, we 
proved an essential uniqueness condition for one PARALIND component matrix. As [22], we used Kruskal's Permutation Lemma [26] to obtain our result. We extended our approach by proving a partial uniqueness condition for one PARALIND component matrix. For this, we made use of the Equivalence Lemma for Partitioned Matrices proven by De Lathauwer [11].

We showed that our PARALIND uniqueness results can also be useful for the study of uniqueness of Tucker3 models with a constrained core array. That is, we provide tools for a systematic analysis of the latter, where so far only ad hoc arguments are used. Conversely, some uniqueness results for constrained Tucker3 models are also applicable to PARALIND and CONFAC. In particular, the Tucker3 uniqueness result of Kiers et al. [23] shows that our PARALIND essential uniqueness condition is not necessary.

As a special case of CONFAC, we considered the block decomposition in rank- $(L, L, 1)$ terms. Using the approach of Jiang and Sidiropoulos [22], we showed that our essential uniqueness condition for $\mathbf{C}$ also implies partial uniqueness of the partitioned matrices $\mathbf{A}$ and $\mathbf{B}$.

Our PARALIND uniqueness conditions require solving a nonlinear system of equations in an unknown vector $\mathbf{d}$. In one particular case (see Lemma 4.4) we have obtained an easy-to-check equivalent condition that does not involve the vector $\mathbf{d}$. A subject of future research would be to eliminate the vector $\mathbf{d}$ from the uniqueness conditions for a larger variety of PARALIND models, in the spirit of studies of CP uniqueness carried out by Jiang and Sidiropoulos [22], De Lathauwer [10], Stegeman et al. [37], and Stegeman [36].

Our uniqueness conditions are formulated for one component matrix only and ignore the relations between the column permutations and scalings or the column-block permutations and scalings for different component matrices that are essentially or partially unique. However, as mentioned in Section 1, in signal processing applications one is usually interested in the uniqueness properties of one component matrix only. Hence, our approach has immediate pratical implications. Still, we conjecture that uniqueness results for three component matrices together are possible to obtain, possibly inspired by the proof of Kruskal's [26] uniqueness condition for CP; see Stegeman and Sidiropoulos [38].

Finally, we would like to point out that also other types of uniqueness occur in PARALIND and CONFAC models and constrained Tucker3 models, than the essential uniqueness or the partial uniqueness defined in Definitions 3.1 and 3.2, respectively. As an example, consider the PARALIND model with constraint matrices (5.6). Numerical experiments show that alternatives for $\mathbf{C}=\mathbf{I}_{4}$ are 
of the form

$$
\mathbf{C}=\left[\begin{array}{llll}
* & 0 & 0 & 0 \\
0 & * & 0 & 0 \\
0 & 0 & * & 0 \\
* & * & * & *
\end{array}\right],
$$

where $*$ denotes a nonzero element. Hence, only the last column of $\mathbf{C}$ appears to be identified up to scaling (or: only the first three rows are identified). This is not partial uniqueness according to Definition 3.2. Analogous examples have been encountered for constrained Tucker3 models, see e.g. Ten Berge and Smilde [41]. It would require a more detailed study of CONFAC and PARALIND models to prove systematic uniqueness conditions for this type of uniqueness. 


\section{Appendix: proofs}

Proof of Theorem 6.2. The result follows if we show that the condition of the Equivalence Lemma for Partitioned Matrices holds for the partitioned matrices $\mathbf{A} \boldsymbol{\Pi}_{a}$ and $\overline{\mathbf{A}} \boldsymbol{\Pi}_{a}$. As in the proof of $(i)$ of Proposition 3.3, the conditions of the theorem imply that $\overline{\mathbf{A}}$ has full column rank. This implies $k_{\overline{\mathbf{A}}}^{\prime}=F$, and the condition of the Equivalence Lemma for Partitioned Matrices becomes: for any vector $\mathbf{x}$ such that $\omega^{\prime}\left(\boldsymbol{\Pi}_{a}^{T} \overline{\mathbf{A}}^{T} \mathbf{x}\right) \leq 1$, we have $\omega^{\prime}\left(\boldsymbol{\Pi}_{a}^{T} \mathbf{A}^{T} \mathbf{x}\right) \leq \omega^{\prime}\left(\boldsymbol{\Pi}_{a}^{T} \overline{\mathbf{A}}^{T} \mathbf{x}\right)$. For any $\mathbf{x}$, we have

$$
(\mathbf{B} \otimes \mathbf{C}) \mathbf{G}_{1} \boldsymbol{\Pi}_{a}\left(\mathbf{A} \boldsymbol{\Pi}_{a}\right)^{T} \mathbf{x}=(\overline{\mathbf{B}} \otimes \overline{\mathbf{C}}) \mathbf{G}_{1} \boldsymbol{\Pi}_{a}\left(\overline{\mathbf{A}} \boldsymbol{\Pi}_{a}\right)^{T} \mathbf{x} .
$$

Suppose $\omega^{\prime}\left(\boldsymbol{\Pi}_{a}^{T} \overline{\mathbf{A}}^{T} \mathbf{x}\right)=0$. Then the right-hand side of (A.1) equals the all-zero vector. Since $(\mathbf{B} \otimes \mathbf{C}) \mathbf{G}_{1} \boldsymbol{\Pi}_{a}$ has full column rank, it follows that also $\left(\mathbf{A} \boldsymbol{\Pi}_{a}\right)^{T} \mathbf{x}$ is all-zero. Hence, $\omega^{\prime}\left(\boldsymbol{\Pi}_{a}^{T} \overline{\mathbf{A}}^{T} \mathbf{x}\right)=$ 0 implies $\omega^{\prime}\left(\boldsymbol{\Pi}_{a}^{T} \mathbf{A}^{T} \mathbf{x}\right)=0$.

It remains to show that $\omega^{\prime}\left(\boldsymbol{\Pi}_{a}^{T} \overline{\mathbf{A}}^{T} \mathbf{x}\right)=1$ implies $\omega^{\prime}\left(\boldsymbol{\Pi}_{a}^{T} \mathbf{A}^{T} \mathbf{x}\right) \leq 1$ Rewriting (A.1) in $J \times K$ matrix form, we obtain

$$
\mathbf{B} \boldsymbol{\Phi} \operatorname{diag}\left(\boldsymbol{\Psi}^{T} \boldsymbol{\Pi}_{a} \boldsymbol{\Pi}_{a}^{T} \mathbf{A}^{T} \mathbf{x}\right) \boldsymbol{\Omega}^{T} \mathbf{C}^{T}=\overline{\mathbf{B}} \boldsymbol{\Phi} \operatorname{diag}\left(\mathbf{\Psi}^{T} \boldsymbol{\Pi}_{a} \boldsymbol{\Pi}_{a}^{T} \overline{\mathbf{A}}^{T} \mathbf{x}\right) \boldsymbol{\Omega}^{T} \overline{\mathbf{C}}^{T} .
$$

Suppose $\omega^{\prime}\left(\boldsymbol{\Pi}_{a}^{T} \overline{\mathbf{A}}^{T} \mathbf{x}\right)=1$. Then $\boldsymbol{\Psi}^{T} \boldsymbol{\Pi}_{a} \boldsymbol{\Pi}_{a}^{T} \overline{\mathbf{A}}^{T} \mathbf{x}$ is equal to $\boldsymbol{\Psi}^{T} \boldsymbol{\Pi}_{a} \mathbf{g}_{f}$ for some vector $\mathbf{g}_{f}$ with the same partition as $\overline{\mathbf{A}} \boldsymbol{\Pi}_{a}$ and nonzero elements only in some part $f$. From (A.2), it follows that

$$
\begin{aligned}
\operatorname{rank}\left(\mathbf{B} \boldsymbol{\Phi} \operatorname{diag}\left(\boldsymbol{\Psi}^{T} \boldsymbol{\Pi}_{a} \boldsymbol{\Pi}_{a}^{T} \mathbf{A}^{T} \mathbf{x}\right) \boldsymbol{\Omega}^{T} \mathbf{C}^{T}\right) & =\operatorname{rank}\left(\overline{\mathbf{B}} \boldsymbol{\Phi} \operatorname{diag}\left(\boldsymbol{\Psi}^{T} \boldsymbol{\Pi}_{a} \boldsymbol{\Pi}_{a}^{T} \overline{\mathbf{A}}^{T} \mathbf{x}\right) \boldsymbol{\Omega}^{T} \overline{\mathbf{C}}^{T}\right) \\
& \leq \operatorname{rank}\left(\boldsymbol{\Phi} \operatorname{diag}\left(\boldsymbol{\Psi}^{T} \boldsymbol{\Pi}_{a} \boldsymbol{\Pi}_{a}^{T} \overline{\mathbf{A}}^{T} \mathbf{x}\right) \boldsymbol{\Omega}^{T}\right) \\
& \leq \max _{f=1, \ldots, F} \max _{\mathbf{g}_{f}}\left(\operatorname{rank}\left(\boldsymbol{\Phi} \operatorname{diag}\left(\mathbf{\Psi}^{T} \boldsymbol{\Pi}_{a} \mathbf{g}_{f}\right) \boldsymbol{\Omega}^{T}\right)\right) \\
& =N^{* *} .
\end{aligned}
$$

Let $\mathbf{d}=\boldsymbol{\Pi}_{a}^{T} \mathbf{A}^{T} \mathbf{x}$. If (A.3) implies $\omega^{\prime}(\mathbf{d}) \leq 1$, then the condition of the Equivalence Lemma for Partitioned Matrices holds. The proof is complete by observing that this is exactly condition (6.2).

Proof of Proposition 9.1. We define

$$
\mathbf{M}_{\mathbf{A}, \mathbf{B}}=\left[\left(\mathbf{A}_{1} \odot \mathbf{B}_{1}\right) \mathbf{1}_{L} \cdots\left(\mathbf{A}_{R} \odot \mathbf{B}_{R}\right) \mathbf{1}_{L}\right] .
$$

The structured part of the $I J \times K$ matricized block decomposition in rank- $(L, L, 1)$ terms equals $\mathbf{M}_{\mathbf{A}, \mathbf{B}} \mathbf{C}^{T}$; see [12]. Suppose we have an alternative decomposition $(\overline{\mathbf{A}}, \overline{\mathbf{B}}, \overline{\mathbf{C}})$ and $\overline{\mathbf{C}}=\mathbf{C} \boldsymbol{\Pi} \boldsymbol{\Lambda}$, where $\boldsymbol{\Pi}$ is a permutation matrix and $\boldsymbol{\Lambda}$ is a nonsingular diagonal matrix. We have

$$
\mathbf{M}_{\mathbf{A}, \mathbf{B}} \mathbf{C}^{T}=\mathbf{M}_{\overline{\mathbf{A}}, \overline{\mathrm{B}}} \overline{\mathbf{C}}^{T}=\mathbf{M}_{\overline{\mathbf{A}}, \overline{\mathrm{B}}} \boldsymbol{\Lambda} \boldsymbol{\Pi}^{T} \mathbf{C}^{T}
$$


Since $\mathbf{C}$ has full column rank, it follows that $\mathbf{M}_{\overline{\mathbf{A}}, \overline{\mathbf{B}}}=\mathbf{M}_{\mathbf{A}, \mathbf{B}} \boldsymbol{\Pi} \boldsymbol{\Lambda}^{-1}$. Hence, $\mathbf{M}_{\overline{\mathbf{A}}, \overline{\mathbf{B}}}$ contains the reordered and rescaled columns of $\mathbf{M}_{\mathbf{A}, \mathbf{B}}$. Suppose $\left(\overline{\mathbf{A}}_{1} \odot \overline{\mathbf{B}}_{1}\right) \mathbf{1}_{L}=\lambda\left(\mathbf{A}_{2} \odot \mathbf{B}_{2}\right) \mathbf{1}_{L}$. Then $\overline{\mathbf{A}}_{1} \overline{\mathbf{B}}_{1}^{T}=\lambda \mathbf{A}_{2} \mathbf{B}_{2}^{T}$. Since $\operatorname{rank}\left(\mathbf{A}_{f} \mathbf{B}_{f}^{T}\right)=L$, the column spaces of $\overline{\mathbf{A}}_{1}$ and $\mathbf{A}_{2}$ are identical, and the column spaces of $\overline{\mathbf{B}}_{1}$ and $\mathbf{B}_{2}$ are identical. This completes the proof.

Proof of Proposition 9.2. We start by proving the following necessary and sufficient condition for uniqueness:

$$
\mathbf{M}_{\mathbf{A}, \mathbf{B}} \mathbf{d}=(\mathbf{F} \odot \mathbf{G}) \mathbf{1}_{L} \quad \text { for some } \mathbf{F}(I \times L) \text { and } \mathbf{G}(J \times L) \quad \text { implies } \quad \omega(\mathbf{d}) \leq 1 .
$$

Condition (A.6) is analogous to Condition A for CP in [22]. First, we show show sufficiency. It follows from Proposition 9.1 that we only need to show essential uniqueness of C. Suppose we have an alternative decomposition $(\overline{\mathbf{A}}, \overline{\mathbf{B}}, \overline{\mathbf{C}})$. From the Permutation Lemma (Lemma 4.1) and $\mathbf{C}$ full column rank, it follows that we need to show that $\omega\left(\mathbf{C}^{T} \mathbf{x}\right) \leq \omega\left(\overline{\mathbf{C}}^{T} \mathbf{x}\right)$ for all $\mathbf{x}$ with $\omega\left(\overline{\mathbf{C}}^{T} \mathbf{x}\right) \leq 1$. We have $\mathbf{M}_{\mathbf{A}, \mathbf{B}} \mathbf{C}^{T} \mathbf{x}=\mathbf{M}_{\overline{\mathbf{A}}, \overline{\mathbf{B}}} \overline{\mathbf{C}}^{T} \mathbf{x}$. Condition (A.6) implies that $\mathbf{M}_{\mathbf{A}, \mathbf{B}}$ has full column rank. Indeed, since $\mathbf{M}_{\mathbf{A}, \mathbf{B}}$ does not contain all-zero columns (the $I \times J$ matrix form of column $f$ equals $\mathbf{A}_{f} \mathbf{B}_{f}^{T}$, which has rank $L$ ), a rank deficient matrix would imply a linear combination of at least two columns constituting the all-zero vector, and the latter can be written as $(\mathbf{F} \odot \mathbf{G}) \mathbf{1}_{L}$ with $\mathbf{F}$ all-zero. Since $\mathbf{M}_{\mathbf{A}, \mathbf{B}}$ has full column rank, $\omega\left(\overline{\mathbf{C}}^{T} \mathbf{x}\right)=0$ implies $\omega\left(\mathbf{C}^{T} \mathbf{x}\right)=0$. Next, suppose $\omega\left(\overline{\mathbf{C}}^{T} \mathbf{x}\right)=1$. For $\mathbf{d}=\overline{\mathbf{C}}^{T} \mathbf{x}$, condition (A.6) implies that $\omega(\mathbf{d}) \leq 1$. Therefore, the condition of the Permutation Lemma holds and $\mathbf{C}$ is essentially unique.

Next, we show necessity of (A.6). Without loss of generality, we set $\mathbf{C}=\mathbf{I}_{F}$ (see Ten Berge and Sidiropoulos [40]). Suppose condition (A.6) does not hold. In particular, we assume that $\left(\mathbf{A}_{1} \odot \mathbf{B}_{1}\right) \mathbf{1}_{L}+\left(\mathbf{A}_{2} \odot \mathbf{B}_{2}\right) \mathbf{1}_{L}=(\mathbf{F} \odot \mathbf{G}) \mathbf{1}_{L}$ (the general proof is analogous). Then

$$
\mathbf{M}_{\mathbf{A}, \mathbf{B}} \mathbf{I}_{F}=\left[(\mathbf{F} \odot \mathbf{G}) \mathbf{1}_{L}\left(\mathbf{A}_{2} \odot \mathbf{B}_{2}\right) \mathbf{1}_{L} \cdots\left(\mathbf{A}_{F} \odot \mathbf{B}_{F}\right) \mathbf{1}_{L}\right]\left[\begin{array}{ccc}
1 & 0 & \mathbf{0}^{T} \\
-1 & 1 & \mathbf{0}^{T} \\
\mathbf{0} & \mathbf{0} & \mathbf{I}_{F-2}
\end{array}\right],
$$

where $\mathbf{0}$ is the $(F-2) \times 1$ all-zero vector. Hence, we obtain an alternative decomposition that differs from $(\mathbf{A}, \mathbf{B}, \mathbf{C})$. This completes the proof of the necessary and sufficient condition (A.6).

It remains to show that (A.6) is equivalent to condition (9.1). This can be seen as follows. The $I \times J$ matrix form of $\mathbf{M}_{\mathbf{A}, \mathbf{B}} \mathbf{d}=(\mathbf{F} \odot \mathbf{G}) \mathbf{1}_{L}$ is $d_{1} \mathbf{A}_{1} \mathbf{B}_{1}^{T}+\cdots+d_{F} \mathbf{A}_{F} \mathbf{B}_{F}^{T}=\mathbf{F} \mathbf{G}^{T}$, where the rank of the latter is at most $L$. Since any $I \times J$ matrix of at most $\operatorname{rank} L$ can be written as $\mathbf{F} \mathbf{G}^{T}$, conditions (A.6) and (9.1) are equivalent. This completes the proof. 


\section{References}

[1] A.L.F. De Almeida, G. Favier, \& J.C.M. Mota (2006) Space-time multiplexing codes: A tensor modeling approach. In Proc. IEEE SPAWC, Cannes, France, July 2006.

[2] A.L.F. de Almeida, G.Favier, \& J.C.M. Mota (2008) Constrained tensor modeling approach to blind multiple-antenna CDMA schemes. IEEE Transactions on Signal Processing, 56, 2417-2428.

[3] A.L.F. de Almeida, G. Favier, \& J.C.M. Mota (2008) A constrained factor decomposition with application to MIMO antenna systems. IEEE Transactions on Signal Processing, 56, $2429-2442$.

[4] R. Bro (1997) Parafac. Tutorial \& Applications. Chemometrics and Intelligent Laboratory Systems, 38, 149-171.

[5] R. Bro (1998) Multi-way Analysis in the Food Industry: Models, Algorithms and Applications, Ph.D. dissertation, University of Amsterdam, The Netherlands.

[6] R. Bro, R.A. Harshman, N.D. Sidiropoulos, \& M.E. Lundy (2009) Modeling multi-way data with linearly dependent loadings. Journal of Chemometrics, 23, 324-340.

[7] J.D. Carroll \& J.J. Chang (1970) Analysis of individual differences in multidimensional scaling via an $n$-way generalization of Eckart-Young decomposition. Psychometrika, 35, 283319 .

[8] P. Comon (1994) Independent component analysis, a new concept? Signal Processing, 36, 287-314.

[9] L. De Lathauwer, B. De Moor, \& J. Vandewalle (2000). An introduction to independent component analysis. Journal of Chemometrics, 14, 123-149.

[10] L. De Lathauwer (2006) A link between the canonical decomposition in multilinear algebra and simultaneous matrix diagonalization. SIAM Journal on Matrix Analysis and Applications, 28, 642-666.

[11] L. De Lathauwer (2008) Decompositions of a higher-order tensor in block terms - Part I: Lemmas for partitioned matrices. SIAM Journal on Matrix Analysis and Applications, 30, $1022-1032$.

[12] L. De Lathauwer (2008) Decompositions of a higher-order tensor in block terms - Part II: Definitions and uniqueness. SIAM Journal on Matrix Analysis and Applications, 30, 10331066 .

[13] L. De Lathauwer \& J. Castaing (2007) Tensor-based techniques for the blind separation of DS-CDMA signals. Signal Processing, 87, 322-336.

[14] L. De Lathauwer \& A. De Baynast (2008) Blind deconvolution of DS-CDMA signals by means of decomposition in rank- $(1, L, L)$ terms. IEEE Transactions on Signal Processing, 56(4), 1562-1571. 
[15] V. De Silva, \& L.-H. Lim (2008) Tensor rank and the ill-posedness of the best low-rank approximation problem. SIAM Journal on Matrix Analysis and Applications, 30, 1084-1127.

[16] R.A. Harshman (1970) Foundations of the Parafac procedure: models and conditions for an "explanatory" multimodal factor analysis. UCLA Working Papers in Phonetics, 16, 1-84.

[17] R.A. Harshman \& M.E. Lundy (1996) Uniqueness proof for a family of models sharing features of Tucker's three-mode factor analysis and PARAFAC/CANDECOMP. Psychometrika, 61, 133-154.

[18] F.L. HiтchCOCK (1927) The expression of a tensor or a polyadic as a sum of products, Journal of Mathematics and Physics, 6, 164-189.

[19] F.L. Hiтchсоск (1927) Multiple invariants and generalized rank of a $p$-way matrix or tensor, Journal of Mathematics and Physics, 7, 39-70.

[20] P.K. Hopke, P. Pantero, H. Jia, R.T. Ross, \& R.A. Harshman (1998) Three-way (Parafac) factor analysis: examination and comparison of alternative computational methods as applied to ill-conditioned data. Chemometrics and Intelligent Laboratory Systems, 43, 2542.

[21] A. Hyvärinen, J. Karhunen, \& E. Oja (2001) Independent Component Analysis, New York: Wiley.

[22] T. Jiang \& N.D. Sidiropoulos (2004) Kruskal's permutation lemma and the identification of Candecomp/Parafac and bilinear models with constant modulus constraints. IEEE Transactions on Signal Processing, 52, 2625-2636.

[23] H.A.L. Kiers, J.M.F. Ten Berge \& R. Rocci (1997) Uniqueness of three-mode factor models with sparse cores: the $3 \times 3 \times 3$ case. Psychometrika, 62, 349-374.

[24] W.P. Krijnen, T.K. Dijkstra \& A. Stegeman (2008) On the non-existence of optimal solutions and the occurrence of "degeneracy" in the Candecomp/Parafac model. Psychometrika, 73, 431-439.

[25] P.M. Kroonenberg (2008) Applied Multiway Data Analysis, Wiley Series in Probability and Statistics.

[26] J.B. KRuskal (1977) Three-way arrays: rank and uniqueness of trilinear decompositions, with applications to arithmetic complexity and statistics. Linear Algebra and its Applications, 18, 95-138.

[27] D. Nion \& L. De Lathauwer (2007) A tensor-based blind DS-CDMA receiver using simultaneous matrix diagonalization. Proc. VIII IEEE Workshop on Signal Processing Advances in Wireless Communications (SPAWC 2007), June 17-20, 2007, Helsinki, Finland.

[28] N. Sidiropoulos, G. Giannakis, \& R. Bro (2000) Blind Parafac receivers for DS-CDMA systems. IEEE Transactions on Signal Processing, 48, 810-823.

[29] N. Sidiropoulos, R. Bro, \& G. Giannakis (2000) Parallel factor analysis in sensor array processing. IEEE Transactions on Signal Processing, 48, 2377-2388. 
[30] N.D. Sidiropoulos \& G.Z. Dimić (2001) Blind multiuser detection in W-CDMA systems with large delay spread. IEEE Signal Processing Letters, 8, 87-89.

[31] N.D. Sidiropoulos \& R. Budampati (2002) Khatri-Rao space-time codes. IEEE Transactions on Signal Processing, 50, 2377-2388.

[32] A. Smilde, R. Bro, \& P. Geladi (2004) Multi-way Analysis: Applications in the Chemical Sciences. Wiley.

[33] A. Stegeman (2006) Degeneracy in Candecomp/Parafac explained for $p \times p \times 2$ arrays of rank $p+1$ or higher. Psychometrika, 71, 483-501.

[34] A. Stegeman (2007) Degeneracy in Candecomp/Parafac and Indscal explained for several three-sliced arrays with a two-valued typical rank. Psychometrika, 72, 601-619.

[35] A. Stegeman (2008) Low-rank approximation of generic $p \times q \times 2$ arrays and diverging components in the Candecomp/Parafac model. SIAM Journal on Matrix Analysis and Applications, 30, 988-1007.

[36] A. Stegeman (2009) On uniqueness conditions for Candecomp/Parafac and Indscal with full column rank in one mode. Linear Algebra and its Applications, 431, 211-227.

[37] A. Stegeman, J.M.F. Ten Berge, \& L. De Lathauwer (2006) Sufficient conditions for uniqueness in Candecomp/Parafac and Indscal with random component matrices. Psychometrika, 71, 219-229.

[38] A. Stegeman \& N.D. Sidiropoulos (2007) On Kruskal's uniqueness condition for the Candecomp/ Parafac decomposition. Linear Algebra and its Applications, 420, 540-552.

[39] A. Stegeman \& L. De Lathauwer (2009) A method to avoid diverging components in the Candecomp/Parafac model for generic $I \times J \times 2$ arrays. SIAM Journal on Matrix Analysis and Applications, 30, 1614-1638.

[40] J.M.F. Ten Berge \& N.D. Sidiropoulos (2002) On uniqueness in Candecomp/Parafac. Psychometrika, 67, 399-409.

[41] J.M.F. Ten Berge \& A.K. Smilde (2002) Non-triviality and identification of a constrained Tucker3 analysis. Journal of Chemometrics, 16, 609-612.

[42] J.M.F. Ten Berge (2004) Partial uniqueness in Candecomp/Parafac. Journal of Chemometrics, 18, 12-16.

[43] J.M.F. Ten Berge (2004) Simplicity and typical rank of three-way arrays, with applications to Tucker3-analysis with simple cores. Journal of Chemometrics, 18, 17-21.

[44] G. Tomasi \& R. Bro (2006) A Comparison of algorithms for fitting the Parafac model. Computational Statistics \& Data Analysis, 50, 1700-1734.

[45] L.R. TuCKER (1966) Some mathematical notes on three-mode factor analysis. Psychometrika, 31, 279-311. 